Draft VERSion MAY 16, 2018

Typeset using $\mathrm{LAT}_{\mathrm{E}} \mathrm{X}$ twocolumn style in a modified version of AASTeX61

\title{
HUBBLE SPACE TELESCOPE PROPER MOTION (HSTPROMO) CATALOGS OF GALACTIC GLOBULAR CLUSTERS. VI. IMPROVED DATA REDUCTION AND INTERNAL-KINEMATIC ANALYSIS OF NGC 362
}

\author{
Mattia Libralato, ${ }^{1,2,3}$ Andrea Bellini, ${ }^{1}$ Roeland P. van Der Marel, ${ }^{1,4}$ Jay Anderson, ${ }^{1}$ Laura L. Watkins, ${ }^{1}$ \\ Giampaolo Piotto, ${ }^{2,3}$ Francesco R. Ferraro, ${ }^{5,6}$ Domenico Nardiello, ${ }^{2,3}$ and Enrico Vesperini ${ }^{7}$
}

\footnotetext{
${ }^{1}$ Space Telescope Science Institute 3700 San Martin Drive, Baltimore, MD 21218, USA

${ }^{2}$ Dipartimento di Fisica e Astronomia, Università di Padova, Vicolo dell'Osservatorio 3, Padova, I-35122, Italy

${ }^{3}$ INAF-Osservatorio Astronomico di Padova, Vicolo dell'Osservatorio 5, Padova, I-35122, Italy

${ }^{4}$ Center for Astrophysical Sciences, Department of Physics \& Astronomy, Johns Hopkins University, Baltimore, MD 21218, USA

${ }^{5}$ Dipartimento di Fisica e Astronomia, Università di Bologna, Via Gobetti 93/2, Bologna, I-40129, Italy

${ }^{6}$ INAF-Osservatorio Astronomico di Bologna, Via Gobetti 93/3, Bologna, I-40129, Italy

${ }^{7}$ Department of Astronomy, Indiana University, Bloomington, IN 47405, USA
}

(Received March 29, 2018; Revised May 10, 2018; Accepted May 11, 2018)

\begin{abstract}
We present an improved data-reduction technique to obtain high-precision proper motions (PMs) of globular clusters using Hubble Space Telescope data. The new reduction is superior to the one presented in the first paper of this series for the faintest sources in very crowded fields. We choose the globular cluster NGC 362 as a benchmark to test our new procedures. We measure PMs of 117450 sources in the field, showing that we are able to obtain a PM precision better than $10 \mu \mathrm{as} \mathrm{yr}^{-1}$ for bright stars. We make use of this new PM catalog of NGC 362 to study the cluster's internal kinematics. We investigate the velocity-dispersion profiles of the multiple stellar populations hosted by NGC 362 and find new pieces of information on the kinematics of first- and second-generation stars. We analyze the level of energy equipartition of the cluster and find direct evidence for its post-core-collapsed state from kinematic arguments alone. We refine the dynamical mass of the blue stragglers and study possible kinematic differences between blue stragglers formed by collisions and mass transfer. We also measure no significant cluster rotation in the plane of the sky. Finally, we measure the absolute PM of NGC 362 and of the background stars belonging to the Small Magellanic Cloud, finding a good agreement with previous estimates in the literature. We make the PM catalog publicly available.
\end{abstract}

Keywords: globular clusters: individual (NGC 362) - proper motions - stars: kinematics and dynamics - stars: Population II - techniques: photometric

\section{INTRODUCTION}

High-precision proper motions (PMs) have proven to be the most effective tool to analyze the internal kinematics and dynamics of globular clusters (GCs). Although new instruments and missions have been recently developed in this context, the Hubble Space Telescope $(H S T)$ is still the reference astrometric tool for such investigations.

Corresponding author: Mattia Libralato libra@stsci.edu
Bellini et al. (2014, Paper I) computed the PM of stars in 22 GCs using archival HST data sets. These PM catalogs, which represent the state-of-the-art for astrometry, make it possible to investigate a broad range of kinematic studies. For example, Watkins et al. (2015, Paper II) and Watkins et al. (2015, Paper III) used these PMs to compute the velocity dispersions for cluster stars at different radial distances, two-dimensional velocitydispersion spatial maps, dynamical distances and massto-light ratios for these GCs. Furthermore, by means of the same PM catalogs, Baldwin et al. (2016, Paper IV) computed the dynamical mass of the blue stragglers (BSs). 
These catalogs collect the astrometric and photometric data for all objects in the observed fields of these GCs. As such, foreground/background sources are measured with the same astrometric precision as GC stars and can be adopted to infer the cluster's rotation in the plane of the sky of the GCs, as was done by Bellini et al. (2017d, Paper V) for NGC 104 (47 Tuc).

In this paper of the series, we derive a new PM catalog for the GC NGC 362 by using a revised data-reduction strategy, which was specifically designed to improve PM precision and completeness in very-crowded fields. Since the procedures are somewhat complicated, we describe them in detail in the Appendices.

In Appendix A we describe the photometric procedures. A one-pass photometric routine is run to measure the bright, easy to measure stars, and these measurements are used to determine the photometric and astrometric transformations between each exposure and the reference frame. We then introduce a second stage of reduction that (i) simultaneously employs all images at once, and (ii) applies neighbor subtraction. The former feature works best in enhancing the contribution of faint sources otherwise lost in the noise of the images. The latter is particularly effective near the center of GCs. The core of NGC 362 is extremely crowded, thus being a perfect benchmark to highlight the improvements made possible by the new data-reduction strategy.

In Appendix B describe how we compute the relative proper motions for all the stars.

NGC 362 is a core-collapsed GC (e.g., Trager et al. 1995) that is known to host multiple stellar populations (hereafter mPOPs, see, e.g., Piotto et al. 2012; Carretta et al. 2013; Lim et al. 2016; Piotto et al. 2015; Milone et al. 2017), as well as two sequences of BSs (Dalessandro et al. 2013). We investigate the internal kinematics of this GC, in particular of its mPOPs, the level of energy equipartition, and, for the first time, its postcore-collapsed state thanks solely to internal-kinematic arguments (Sect. 3).

Finally, NGC 362 is located in front of the Small Magellanic Cloud (SMC). As such, we can estimate the rotation of NGC 362 in the plane of the sky using the same method described in Paper V.

\section{DATA SETS AND REDUCTION}

We made use of all suitable HST images covering the central field of the cluster ${ }^{1}$. We focused on HighResolution Channel (HRC) and the Wide-Field Channel (WFC) exposures taken with the Advanced Camera for

\footnotetext{
${ }^{1}(\alpha, \delta)_{\mathrm{J} 2000}=\left(01^{\mathrm{h}} 03^{\mathrm{m}} 14^{\mathrm{s}} \cdot 26,-70^{\circ} 50^{\prime} 55^{\prime \prime} \cdot 6\right)$, Goldsbury et al. (2010).
}

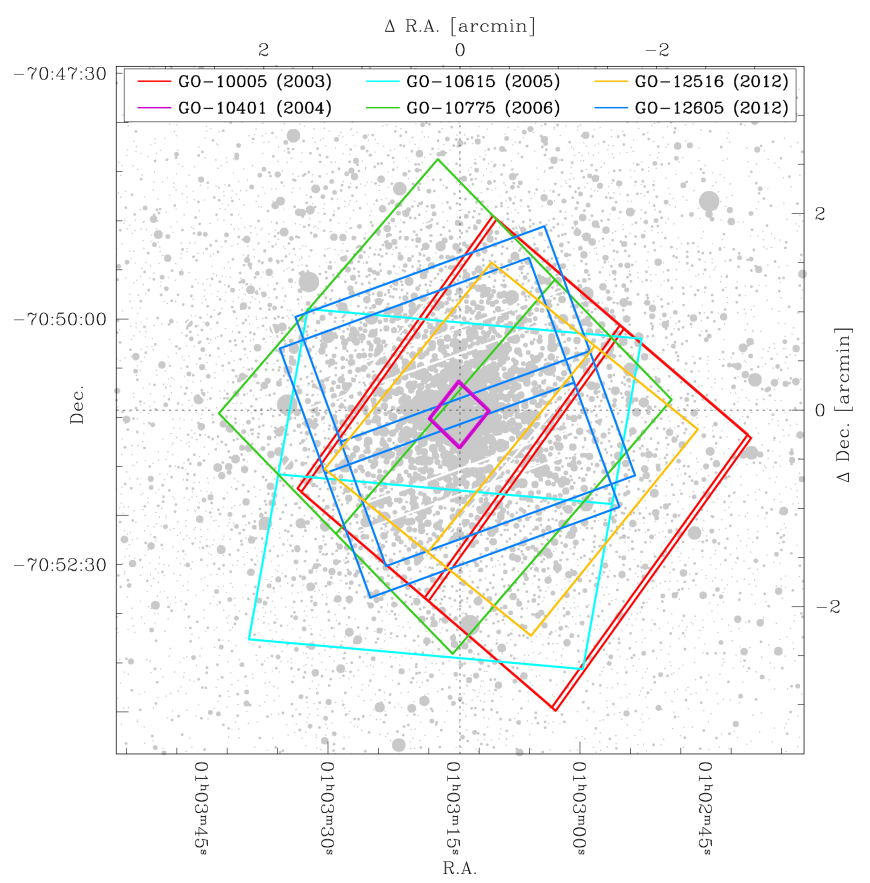

Figure 1. Outline of the FoV covered by the observations used for the analysis of NGC 362. Each footprint is colorcoded according to the GO proposal number. The stellar map is obtained using the $G$-band photometry of the Gaia Data Release 1 (DR1, Gaia Collaboration et al. 2016a,b). Top and right axes are in arcmin with respect to the cluster center given by Goldsbury et al. (2010).

Survey (ACS), and with the Ultraviolet-VISible (UVIS) channel of the Wide-Field Camera 3 (WFC3). Observations of the Wide-Field Planetary Camera 2 (WFPC2) were not used because of the much lower astrometric precision with respect to that of ACS and WFC3 (UVIS) imagers. An overview of the field of view (FoV) covered is shown in Fig. 1. Table 1 lists all the observations analyzed in this paper ${ }^{2}$.

The data reduction was performed closely following the prescriptions extensively described in Bellini et al. (2017a, 2018) and it is the end result of first- and secondpass photometric reductions. PMs were computed as described in Paper I and Bellini et al. (2018), an advanced evolution of the so-called central overlap method (Eichhorn \& Jefferys 1971). An overview of the procedures adopted and a comparison with the original PM catalog of Paper I are presented in Appendices A and B.

The final PM catalog contains 117450 sources. An overview of the PM catalog is shown in Fig. 2. In panel (a) we present the color-magnitude diagram (CMD) of NGC 362. We were able to measure PMs from the redgiant branch $(\mathrm{RGB})$ down to $\sim 8$ magnitudes below the

2 DOI reference: [10.17909/T9CH53] 
AASTEX HSTPROMO VI. Improved DATA REDUCtion AND INTERNAL-KINEMATIC ANALYSIS OF NGC 3623

Table 1. List of observations of NGC 362 used in this paper.

\begin{tabular}{|c|c|c|c|c|c|}
\hline GO & PI & Instrument/Camera & Filter & $N \times$ Exp. Time & Epoch \\
\hline \multirow[t]{3}{*}{10005} & Lewin & $\mathrm{ACS} / \mathrm{WFC}$ & $\mathrm{F} 435 \mathrm{~W}$ & $4 \times 340 \mathrm{~s}$ & 2003 December \\
\hline & & & F625W & $2 \times 110 \mathrm{~s}, 2 \times 120 \mathrm{~s}$ & \\
\hline & & & F658N & $2 \times 440 \mathrm{~s}, 2 \times 500 \mathrm{~s}$ & \\
\hline 10401 & Chandar & ACS/HRC & F435W & $16 \times 85 \mathrm{~s}$ & 2004 December \\
\hline 10615 & Anderson & $\mathrm{ACS} / \mathrm{WFC}$ & F435W & $30 \times 340 \mathrm{~s}, 5 \times 70 \mathrm{~s}$ & 2005 September \\
\hline \multirow[t]{2}{*}{10775} & Sarajedini & $\mathrm{ACS} / \mathrm{WFC}$ & F606W & $4 \times 150 \mathrm{~s}, 1 \times 10 \mathrm{~s}$ & 2006 June \\
\hline & & & F814W & $4 \times 170 \mathrm{~s}, 1 \times 10 \mathrm{~s}$ & \\
\hline \multirow[t]{5}{*}{12516} & Ferraro & WFC3/UVIS & F390W & $14 \times 348 \mathrm{~s}$ & 2012 April \\
\hline & & & F555W & $1 \times 160 \mathrm{~s}, 1 \times 200 \mathrm{~s}$ & \\
\hline & & & & $1 \times 144 \mathrm{~s}, 1 \times 145 \mathrm{~s}$ & \\
\hline & & & & $6 \times 150 \mathrm{~s}$ & \\
\hline & & & F814W & $12 \times 348 \mathrm{~s}, 3 \times 390 \mathrm{~s}$ & \\
\hline \multirow[t]{3}{*}{12605} & Piotto & WFC3/UVIS & F275W & $6 \times 519 \mathrm{~s}$ & 2012 September \\
\hline & & & F336W & $4 \times 350 \mathrm{~s}$ & \\
\hline & & & F438W & $4 \times 54 \mathrm{~s}$ & \\
\hline
\end{tabular}

main-sequence (MS) turn-off. In panel (b) we show the FoV covered by our data sets. In all panels, sources are color-coded according to the temporal baseline used to compute their PM (see histogram in panel c). The stellar PMs were computed with a temporal baseline between about 1 and 9 yr. Stars within the half-light radius $\left(r_{\mathrm{h}}=0.82\right.$ arcmin, Harris 1996, 2010 edition) have PMs computed over a $\sim 9$-yr baseline, while outside of $r_{\mathrm{h}}$ the available temporal coverage is shorter. Two groups of stars are clearly distinguishable in the vector-point diagram (VPD) of panel (d): NGC 362 stars are centered at the origin of the VPD, by construction; SMC stars populate the lesser clump around $(-5.9,1.3)$ mas $\mathrm{yr}^{-1}$. In panel (e) we show the 1-D PM error as a function of $m_{\mathrm{F} 606 \mathrm{~W}}$ magnitude. The PM of bright, wellmeasured stars in the deep exposures is precise to better than $10 \mu$ as $\mathrm{yr}^{-13}$. Faint stars at about $m_{\mathrm{F} 606 \mathrm{~W}} \sim 26$

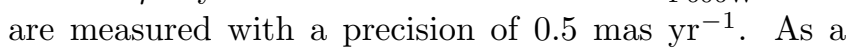
reference, the expected end-of-mission precision of Gaia at $m_{\mathrm{F} 606 \mathrm{~W}} \sim 21.5$ (the Gaia faint limit) is $\sim 1 \mathrm{mas} \mathrm{yr}^{-1}$ (see Pancino et al. 2017); our PM error at this magni-

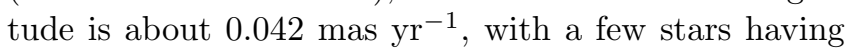

\footnotetext{
3 The effect of the annual parallax can be as large as $\sim 26 \mu$ as $\mathrm{yr}^{-1}$ for stars that are members of NGC 362, a factor two greater than the PM error for well-measured, bright stars. However, we computed the PMs using cluster stars as reference. The parallax effect has no impact on NGC 362 stars but it is instead transferred, with opposite sign, to foreground/background objects. Therefore, the analyses of the internal kinematics of NGC 362 in our paper should not be affected by annual-parallax effects.
}

errors around 0.020 mas $\mathrm{yr}^{-1}$. At the faint end PMs are also measured reasonably well (i.e., $\operatorname{err}_{\mu}<0.5$ mas $\mathrm{yr}^{-1}$ at $\left.m_{\mathrm{F} 606 \mathrm{~W}} \sim 25\right)$, even when a small number of exposures is available. This is thanks to the neighborsubtraction characteristics of KS2 (see Appendix A).

We also made a comparison with the PMs recently available from the Gaia DR2 (Gaia Collaboration et al. 2016a, 2018a). We computed the median 1-D PM error $\operatorname{err}_{\mu}$ as a function of magnitude using only the stars in common between the two catalogs and found that the precision of our PMs is between a factor 4 (bright stars with $G \sim 15.4$ ) and 85 (faint stars with $G>19$ ) times better than that of the Gaia DR2. Furthermore, we found that the $\sim 75 \%$ of the common stars are beyond 1 arcmin from the center, as might be expected because of the level of crowding in the field.

Two sequences of errors are visible in panel (e) of Fig. 2, hereafter the "best" and the "worst" sequences. The worst sequence seems to also be split in two branches, one brighter than $m_{\mathrm{F} 606 \mathrm{~W}} \sim 23$ and one fainter. The faint tail of the worst sequence is related to stars which PM is measured with a temporal baseline lower than 3 yr. Furthermore, the majority of stars belonging to the worst-measured sequences have PMs computed with less than 30 and 50 images, respectively.

Finally, we investigated the presence of positional-, magnitude- and color-dependent systematic errors in our PMs and found no clear trends, giving us assurance that our PMs are not affected by large-scale systematic effects. 

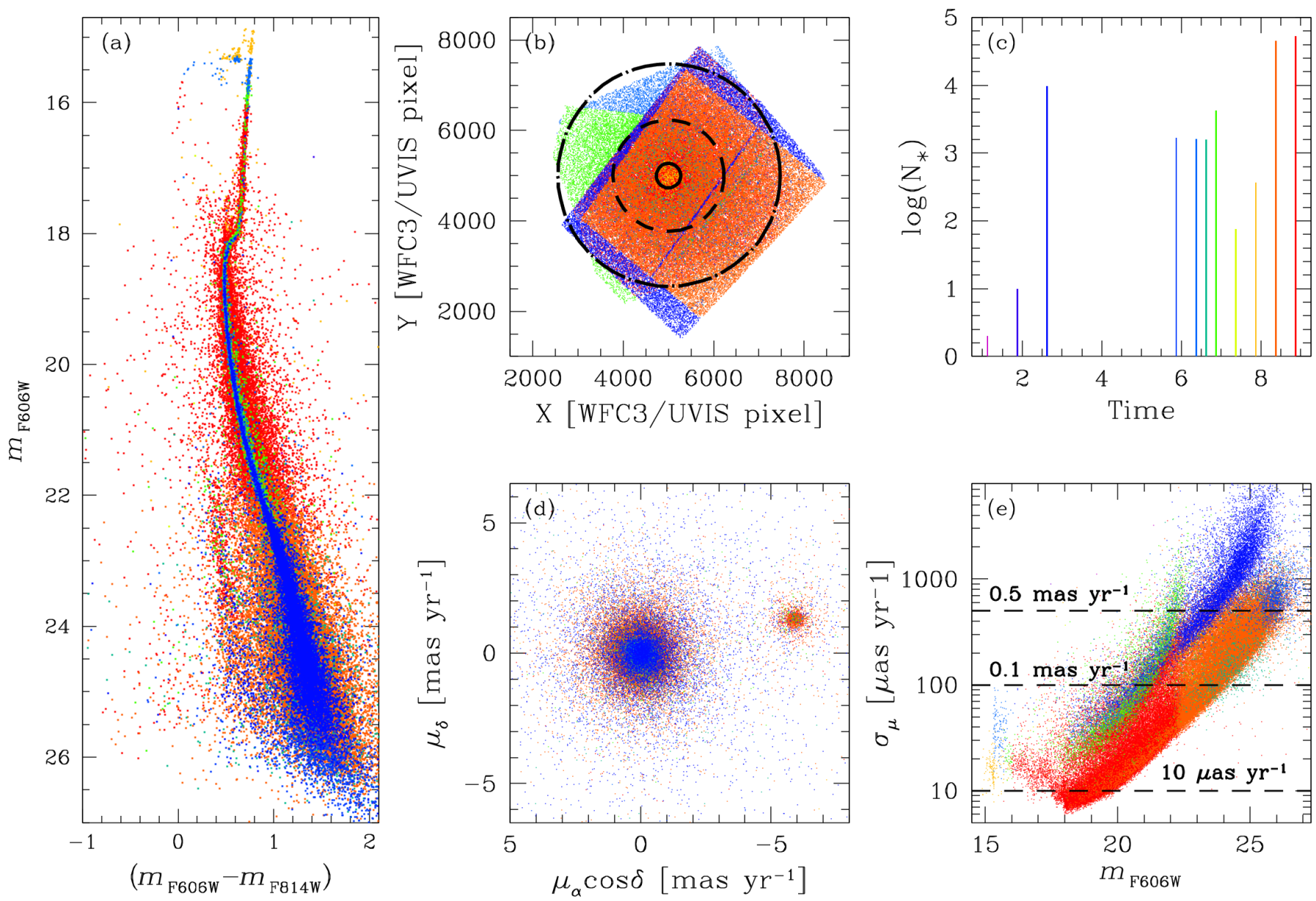

Figure 2. Overview of the PM catalog constructed in this paper. The $m_{\mathrm{F} 606 \mathrm{~W}}$ versus $\left(m_{\mathrm{F} 606 \mathrm{~W}}-m_{\mathrm{F} 814 \mathrm{~W}}\right)$ CMD of NGC 362 is presented in panel (a). In panel (b) we show the field covered by the analyzed data sets in units of WFC3/UVIS pixels. The cluster is centered at $(5000,5000)$ WFC3/UVIS pixels. The solid circle has a radius equal to the $r_{\mathrm{c}}$ of NGC 362 (0.18 arcmin, Harris 1996, 2010 edition), while the short-dashed and the long-dashed circles have a radius equal to $r_{\mathrm{h}}$ and $2 r_{\mathrm{h}}$, respectively. The histogram in panel (c) shows the logarithm of the number of sources as a function of the temporal baseline. The points in all panels of this Figure are color-coded according to the temporal baseline as shown in panel (c). The relative VPD in equatorial coordinates is shown in panel (d). Panel (e) shows the 1-D PM error $\operatorname{err}_{\mu}$ in $\mu$ as $\mathrm{yr}^{-1}$ as a function of the $m_{\mathrm{F} 606 \mathrm{~W}}$

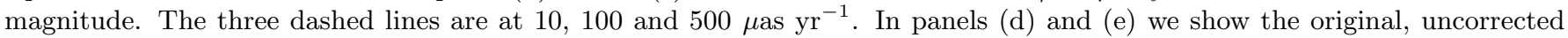
PMs (see the text for details).

\section{ANALYSIS}

\subsection{PM selections and validation}

The analysis of the internal kinematics of GCs requires a careful selection of the best-measured stars. In general, we adopted the following constraints on stars to be considered: (i) the reduced $\chi^{2}$ of the PM fit must be lower than 1.5, (ii) PM-fitting rejection rate must be lower than $15 \%$, and (iii) the quality of fit (QFIT) value (a measure of how well the star is fit by a single-star PSF) must be greater than the $50^{\text {th }}$-percentile QFIT value at the star's magnitude level (see Appendices A and B for a more complete description of these quantities).

In addition, we kept cluster stars with a PM error lower than half the local velocity dispersion $\sigma_{\mu}$ of the closest (in distance and magnitude) 75 cluster stars (see
Sect. 8.3 of Paper I). We considered as cluster members stars with a relative PM lower than 1.2 mas $\mathrm{yr}^{-1}$ (about 5 times the velocity dispersion of faint members of NGC 362).

To analyze how different QFIT cuts could change the inferred velocity-dispersion profile of NGC 362, we computed the value of $\sigma_{\mu}$ in different radial and magnitude bins for QFIT thresholds from the $5^{\text {th }}$ to the $95^{\text {th }}$ percentile, with steps of $5 \%$. We found that the values of $\sigma_{\mu}$ in our tests are all consistent with each other, mainly because most of the outliers are already removed with the reduced $\chi^{2}$ and the rejection-rate selections. As such, we arbitrarily chose the $50^{\text {th }}$ percentile.

In Fig. 3 we show the velocity-dispersion radial profile inferred from the PMs measured in our catalog with dif- 

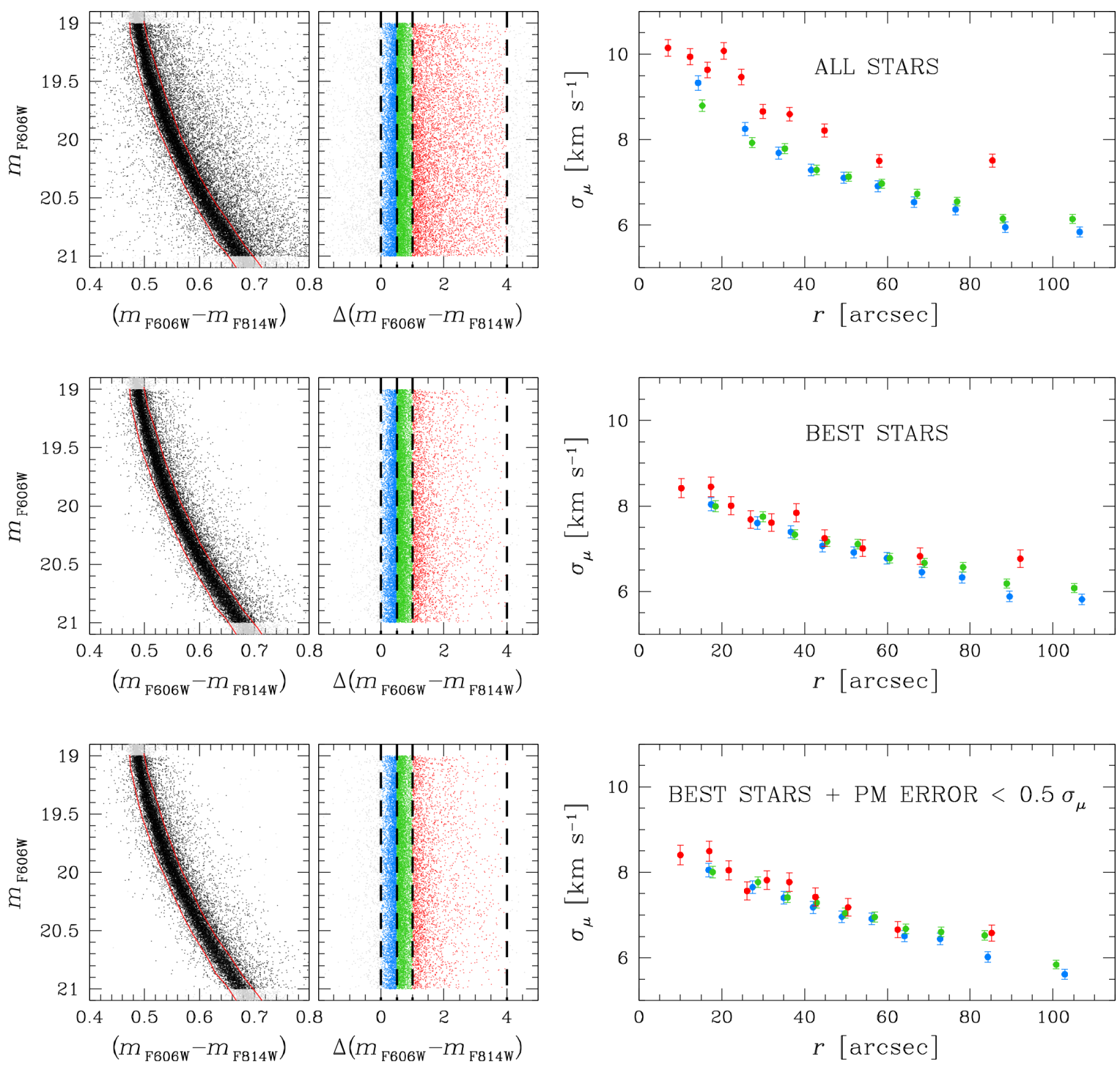

Figure 3. On the left we present the $m_{\mathrm{F} 606 \mathrm{~W}}$ versus $\left(m_{\mathrm{F} 606 \mathrm{~W}}-m_{\mathrm{F} 814 \mathrm{~W}}\right)$ CMD of MS stars of NGC 362 with $19<m_{\mathrm{F} 606 \mathrm{~W}}<21$. The red fiducial lines are used to rectify the CMD (central panels). The dashed, vertical black lines define three samples of stars that are used to compute velocity-dispersion radial profiles. In the right-hand panels, we show the combined velocity-dispersion profiles as a function of distance from the cluster center for these three groups. From top to bottom, we present the analysis obtained by considering all stars, well-measured stars defined as described in the text, and a sub-sample of well-measured stars with PM errors smaller than half of the average velocity dispersion, respectively. See the text for details

ferent selection criteria. We considered only MS members of NGC 362 with $19<m_{\mathrm{F} 606 \mathrm{~W}}<21$. We drew by hand two fiducial lines enclosing the bulk of MS stars and made use of these lines to rectify the MS. We then split the MS in three groups: "blue" stars with $0 \leq \Delta\left(m_{\mathrm{F} 606 \mathrm{~W}}-m_{\mathrm{F} 814 \mathrm{~W}}\right)<0.5$ (azure points), "red" sources with $0.5 \leq \Delta\left(m_{\mathrm{F} 606 \mathrm{~W}}-m_{\mathrm{F} 814 \mathrm{~W}}\right)<1.0$ (green points $)$, and "very-red" objects $1.0 \leq \Delta\left(m_{\mathrm{F} 606 \mathrm{~W}}-\right.$ $\left.m_{\mathrm{F} 814 \mathrm{~W}}\right)<4.0$ (red points). Finally, for each group we computed the velocity dispersion $\sigma_{\mu}$ in 10 equally- populated radial bins ${ }^{4}$. The velocity dispersion is computed as described in van der Marel \& Anderson (2010), i.e., by correcting the observed scatter of the PMs for the uncertainties of the individual PMs.

Without any selection (top panels), very-red objects appear kinematically hotter than blue and red stars. Most of these sources are blends or poorly-measured stars due to crowding, with some contamination due to binaries (the fraction of MS binaries in NGC 362 is less

\footnotetext{
${ }^{4}$ Binning the data will always introduce biases, some of which can be quite subtle. We applied different criteria for the bin sizes and found consistent results within the errors.
} 

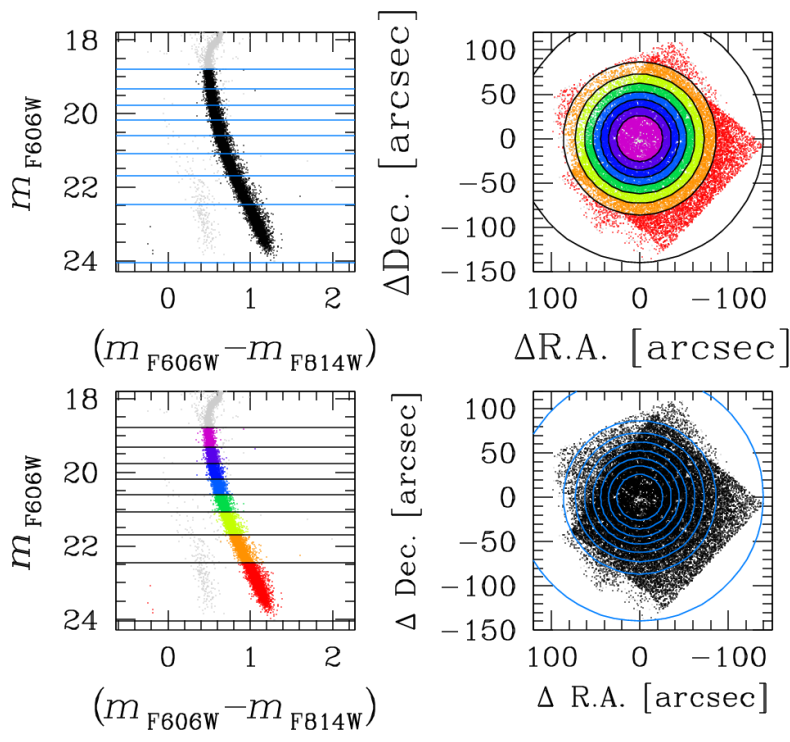
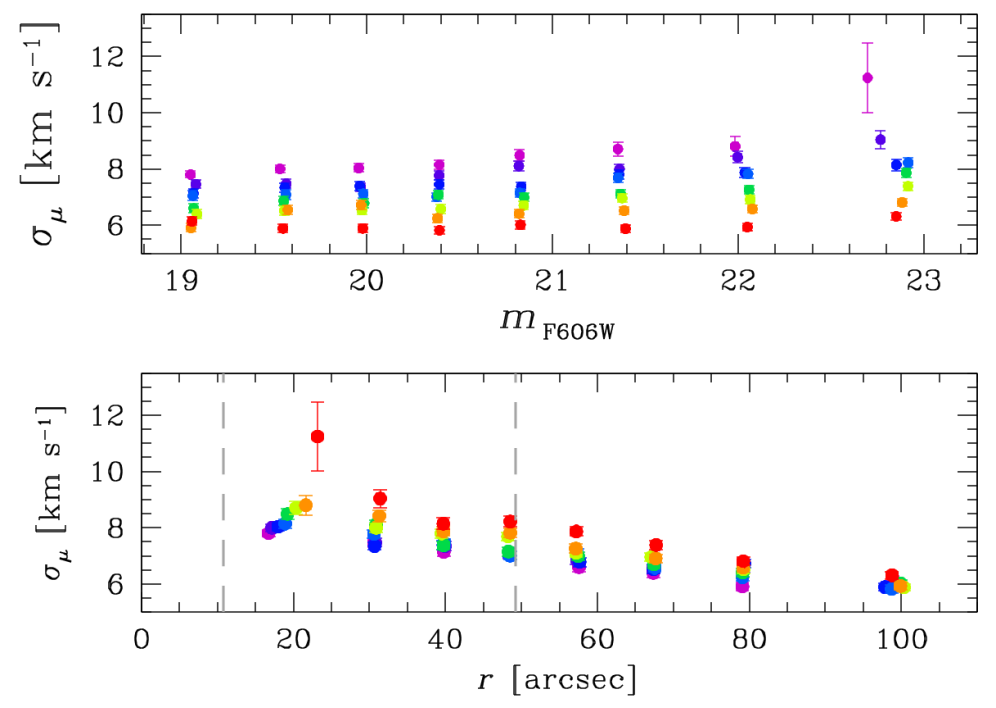

Figure 4. Velocity-dispersion profiles of MS stars of NGC 362. (Top-left): $m_{\mathrm{F} 606 \mathrm{~W}}$ versus $\left(m_{\mathrm{F} 606 \mathrm{~W}}-m_{\mathrm{F} 814 \mathrm{~W}}\right)$ CMD. Black points represent NGC 362 members that survived the quality selections described in the text. Gray dots are non-MS objects. We considered only stars from the MS turn-off $\left(m_{\mathrm{F} 606 \mathrm{~W}} \sim 18.8\right)$ down to five magnitudes below (about the faintest magnitude of the stars in the sample, $\left.m_{\mathrm{F} 606 \mathrm{~W}} \sim 24\right)$. The azure lines define the magnitude-bin limits of 8 equally-populated intervals adopted in the analysis. (Top-middle): FoV of selected stars. The black circles define 8 equally-populated radial bins. Stars are color-coded according to the radial interval they belong to. (Top-right): $\sigma_{\mu}$ as a function of $m_{\mathrm{F} 606 \mathrm{~W}}$. Points (with error bars) are color-coded as in top-middle panel. (Bottom): as on the top panels, but for $\sigma_{\mu}$ in different $m_{\mathrm{F} 606 \mathrm{~W}}$ bins as a function of radial distance.

than 5\%, Milone et al. 2012). As such, a systematic component is present in the PM of these objects, resulting in a faster (by 0.025 mas $\mathrm{yr}^{-1}$ or $\sim 1 \mathrm{~km} \mathrm{~s}^{-1}$ assuming a distance of $8.6 \mathrm{kpc}$, Harris 1996, 2010 edition) motion with respect to blue and red sources.

In the middle panels of Fig. 3, we show the velocitydispersion profile obtained by considering only the best stars defined using the PM $\chi^{2}$, the rejection rate and the F606W/F814W QFIT selections. The agreement between the three groups is now improved, with only a marginal departure at large radii.

Finally, in the bottom panels we present the velocity dispersions of the three groups after we further add a selection on the PM error based on the local value of $\sigma_{\mu}$. By removing additional outliers, the three $\sigma_{\mu}$ trends agree even in the outermost part of the field.

At a given radial distance, faint, less-massive MS stars move faster (are kinematically hotter) than bright, more-massive MS stars in accordance with what we would expect from energy equipartition. In addition, stars of similar mass closer to the center of the cluster are expected to move faster than those far from the center because of hydrostatic equilibrium. To examine these two effects, we selected a sample of MS stars from about the MS turn-off $\left(m_{\mathrm{F} 606 \mathrm{~W}} \sim 18.8\right)$ to five magnitudes below. We then divided the sample in 8 equallypopulated bins in magnitude and radial distance, and computed the combined velocity dispersion in each bin.
In Fig. 4 we summarize the results. In the top panel, the behavior induced by energy equipartition is clear: the brighter the star, the heavier we would expect it to be, and hence the slower its motion. In the bottom panel, stars far from the center of NGC 362 are kinematically colder than those near the center at a given magnitude (mass), as expected from hydrostatic equilibrium.

\section{2. mPOP kinematics}

NGC 362 is known to host mPOPs along its RGB and sub-giant branch (SGB; see e.g., Piotto et al. 2012; Carretta et al. 2013; Lim et al. 2016). In the following analyses, we considered only the best stars from the photometric (using the prescriptions given in Appendix B.1) and astrometric (see Sect. 3.1) points of view. The photometric selections were applied to all F275W, F336W, F438W, F606W and F814W filters, and are used to identify the different mPOPs in the CMD.

\subsubsection{Red-giant branch}

mPOP tagging - Spectroscopic and photometric studies of the RGB stars of NGC 362 have revealed peculiar features, i.e., an anti-correlation in the $\mathrm{Na}-\mathrm{O}$ plane and different $\mathrm{Ba}$ abundances, with Ba-rich stars populating a distinct RGB in the Strömgren $v$ versus $(v-y)$ CMDs (see Carretta et al. 2013, and references therein). Furthermore, Lim et al. (2016) found that CN-weak and 
CN-strong stars populated distinct sequences along the RGB in CMDs based on narrow-band photometry.

More recently, as part of the project "Hubble Space Telescope UV Legacy Survey of Galactic GCs" (Piotto et al. 2015), Milone et al. (2017) analyzed the RGB of 57 Galactic GCs, classifying NGC 362 as a type-II cluster. The RGBs of type-II GCs present (pseudo) twocolor diagrams ("chromosome maps") with split first$(1 \mathrm{G})$ and second-generation $(2 \mathrm{G})$ sequences. Some of the mPOPs of these type-II GCs are also enriched in the total $\mathrm{C}+\mathrm{N}+\mathrm{O}$ abundance, the iron content and the abundance of $s$-process elements (such as $\mathrm{Ba}$ ).

To separate the different mPOPs in NGC 362, we followed the approach of Milone et al. (2017). First, in the $m_{\mathrm{F} 814 \mathrm{~W}}$ versus $\mathrm{c}_{\mathrm{F} 275 \mathrm{~W}, \mathrm{~F} 336 \mathrm{~W}, \mathrm{~F} 438 \mathrm{~W}}=$ $\left(\mathrm{m}_{\mathrm{F} 275 \mathrm{~W}}-\mathrm{m}_{\mathrm{F} 336 \mathrm{~W}}\right)-\left(\mathrm{m}_{\mathrm{F} 336 \mathrm{~W}}-\mathrm{m}_{\mathrm{F} 438 \mathrm{~W}}\right)$ CMD (panel a1 of Fig. 5), we drew by hand two fiducial lines at the blue and red edges of the RGBs. These lines were then used to rectify the RGBs and compute the pseudocolor $\Delta \mathrm{c}_{\mathrm{F} 275 \mathrm{~W}, \mathrm{~F} 336 \mathrm{~W}, \mathrm{~F} 438 \mathrm{~W}}=\left(\mathrm{c}_{\mathrm{F} 275 \mathrm{~W}, \mathrm{~F} 336 \mathrm{~W}, \mathrm{~F} 438 \mathrm{~W}-}\right.$ fiducial $\left._{\text {red }}\right) /\left(\right.$ fiducial $_{\text {blue }}-$ fiducial $\left._{\text {red }}\right)$. We performed the same procedure in the $m_{\mathrm{F} 814 \mathrm{~W}}$ versus $\left(m_{\mathrm{F} 275 \mathrm{~W}}-\right.$ $\left.m_{\mathrm{F} 814 \mathrm{~W}}\right)$ CMD (panel a2) to compute the pseudocolor $\Delta\left(m_{\mathrm{F} 275 \mathrm{~W}}-m_{\mathrm{F} 814 \mathrm{~W}}\right)$. The resulting chromosome map in the $\Delta \mathrm{c}_{\mathrm{F} 275 \mathrm{~W}, \mathrm{~F} 336 \mathrm{~W}, \mathrm{~F} 438 \mathrm{~W}}$ versus $\Delta\left(m_{\mathrm{F} 275 \mathrm{~W}}-m_{\mathrm{F} 814 \mathrm{~W}}\right)$ plane is shown in panel (a3) of Fig. 5.

As described in Milone et al. (2017), 1G stars are expected to be located at $\Delta \mathrm{c}_{\mathrm{F} 275 \mathrm{~W}, \mathrm{~F} 336 \mathrm{~W}, \mathrm{~F} 438 \mathrm{~W}} \sim 0$ (yellow triangles), while the remaining stars belong to the $2 \mathrm{G}$. The red crosses represent the so-called red-RGB stars, stars with a higher $\mathrm{C}+\mathrm{N}+\mathrm{O}, \mathrm{Fe}$ and $s$-processelement abundances than other stars of the same generation. These red-RGB stars are about the $9 \%$ of the total RGB stars in our sample, in agreement with the analysis of Milone et al. (2017). The remaining stars seem to be split in two groups in the chromosome map (azure dots and green squares).

The $1 \mathrm{G} / 2 \mathrm{G}$ nature of these four groups was confirmed by cross-correlating our first-pass photometric catalog (which includes the saturated stars, even though we cannot measure their PMs) with the spectroscopic catalog of of Carretta et al. (2013). We found that the azureand red-RGB stars are $\mathrm{Na}$ rich and $\mathrm{O}$ poor, as expected by $2 \mathrm{G}$ stars, while the green-RGB stars have a chemistry of either $1 \mathrm{G}$ or $2 \mathrm{G}$ stars. Finally, the yellow RGB stars are located in the Na-poor/O-rich region of the plot, as expected by $1 \mathrm{G}$ stars.

Hereafter, we refer to the $1 \mathrm{G}$ yellow-, 2G azure-, $2 \mathrm{G}$ green-, and $2 \mathrm{G}$ red-RGB populations as populations $\mathrm{A}$, $\mathrm{B}, \mathrm{C}$, and $\mathrm{D}$, respectively.
Internal kinematics - In Fig. 5 we present the analysis of the internal kinematics of the mPOPs identified on the RGB of NGC 362. As in Sect. 3.1, we corrected the observed scatter of the PMs for the PM errors. In panel (b1), we show the combined velocity dispersion $\sigma_{\mu}$ as a function of the radial distance. We inferred the value of $\sigma_{\mu}$ in bins of 29,33, 24, and 27 star each for populations A, B, C, and D, respectively. We then chose as a reference population $\mathrm{A}$ and fitted the data with a $3^{\text {rd }}$-order polynomial (black line). In the inset of panel (b1), we depicted the global $\sigma_{\mu}$ of all stars in our FoV as reference. In panels (b2), (b3), and (b4) we show the normalized difference between $\sigma_{\mu}$ of $2 \mathrm{G}$ and $1 \mathrm{G}$ stars. In each panel, black points were computed by using stars within $r_{\mathrm{h}}$ or between $r_{\mathrm{h}} \leq r<2 r_{\mathrm{h}}$. In panels $(\mathrm{c})$ and $(\mathrm{d})$ of Fig. 5, we show the radial $\left(\sigma_{\text {Rad }}\right)$ and tangential $\left(\sigma_{\text {Tan }}\right)$ velocity dispersion as a function of the radial distance and the normalized difference between $2 \mathrm{G}$ and $1 \mathrm{G}$ mPOPs as in panels (b).

We find that $1 \mathrm{G}$ and $2 \mathrm{G}$ stars exhibit the same kinematics. There is only marginal evidence of population B having a lower $\sigma_{\mu}$ than population $\mathrm{A}$ at the $\sim 2.2 \sigma$ level because of a lower $\sigma_{\text {Tan }}$.

We also computed the radial anisotropy profile for these RGB stars. In Fig. 6 we show the ratio $\sigma_{\text {Tan }} / \sigma_{\operatorname{Rad}}$ as a function of distance from the center of NGC 362 for each mPOP on the RGB. In each panel, the horizontal line represents the average trend of the population computed using all stars in the field. The shaded area indicates the $\pm 1 \sigma$ error bars. On average, both $1 \mathrm{G}$ and $2 \mathrm{G}$ stars are consistent with an isotropic system.

The smaller tangential velocity dispersion of the $2 \mathrm{G}$ population $\mathrm{B}$ is similar, although less evident, to the observational findings and theoretical simulations of 47 Tuc (Richer et al. 2013), NGC 2808 (Bellini et al. 2015), and $\omega$ Cen (Bellini et al. 2018). However, the difference between $\sigma_{\text {Rad }}$ and $\sigma_{\text {Tan }}$ in the RGB population B of NGC 362 is not large enough to create a significant radial anisotropy as in the case of NGC 2808 and $\omega$ Cen.

\subsubsection{Sub-giant branch}

Like the RGB, the SGB of NGC 362 is also split in at least 2 main groups (Piotto et al. 2012). The lesspopulated group is clearly separated from the remaining SGB stars in the $m_{\mathrm{F} 814 \mathrm{~W}}$ versus $\left(m_{\mathrm{F} 336 \mathrm{~W}}-m_{\mathrm{F} 814 \mathrm{~W}}\right)$ CMD. As suggested by Milone et al. (2017), this SGB is connected to the red-RGB population D.

We initially identified the stars belonging to the lesspopulated SGB (hereafter, the red SGB) in the $m_{\mathrm{F} 814 \mathrm{~W}}$ versus $\left(m_{\mathrm{F} 336 \mathrm{~W}}-m_{\mathrm{F} 814 \mathrm{~W}}\right)$ CMD. These stars are about $9 \%$ of the SGB stars in our sample. Then, we analyzed in detail the more-populated SGB by constructing 

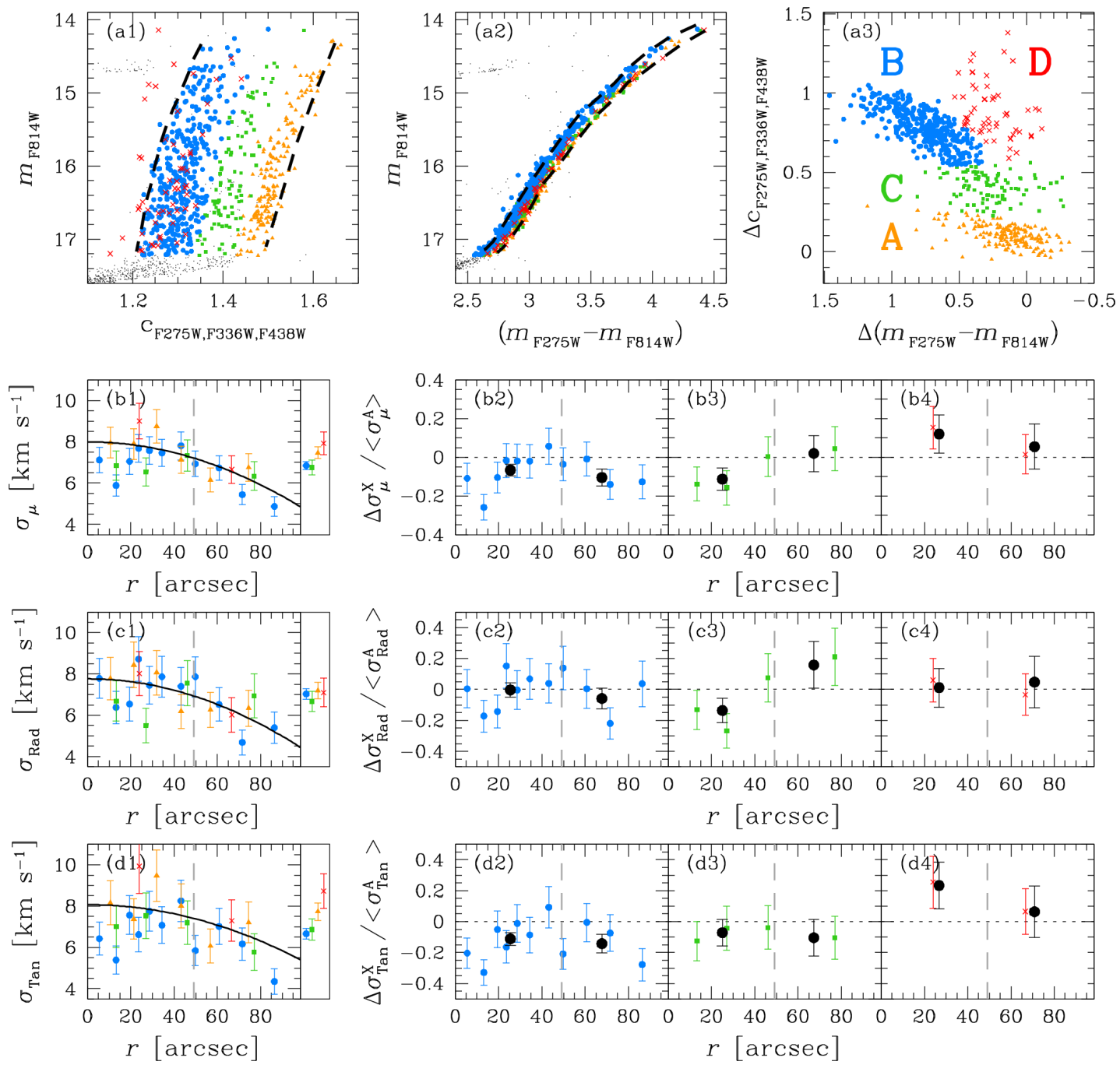

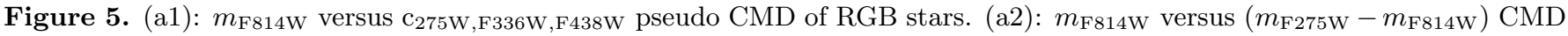
of the same stars. The two black, dashed lines are used to construct the chromosome map. (a3) chromosome map of RGB stars. In these panels, points are color-coded according to the $4 \mathrm{mPOPs} \mathrm{A}$ (yellow triangles), B (azure dots), C (green squares), and $\mathrm{D}$ (red crosses). (b1): $\sigma_{\mu}$ as a function of radial distance. Points are color-coded as in CMDs of panels (a). The solid, black line represents a $3^{\text {rd }}$-order polynomial fit to the population A data (yellow crosses), chosen as a reference. The gray, dashed vertical line marks the half-light radius $\left(r_{\mathrm{h}}\right)$. Points in the inset to the right are the average $\sigma_{\mu}$ over the entire FoV. (b2-b3-b4): normalized difference between $\sigma_{\mu}$ of the $2 \mathrm{G}$ populations with respect to that of the reference $1 \mathrm{G}$ population. The gray, horizontal dashed lines are set at 0 . The two black dots in each panel are the average $\sigma_{\mu}$ for stars with $r<r_{\mathrm{h}}$ and $r_{\mathrm{h}} \leq r<2 r_{\mathrm{h}}$ (dashed, gray vertical line). (c1-c2-c3-c4): As in panels (b) but for the radial velocity dispersion $\sigma_{\text {Rad. }}$ (d1-d2-d3-d4): As in panels (b) and (c) but for the tangential velocity dispersion $\sigma_{\operatorname{Tan}}$.

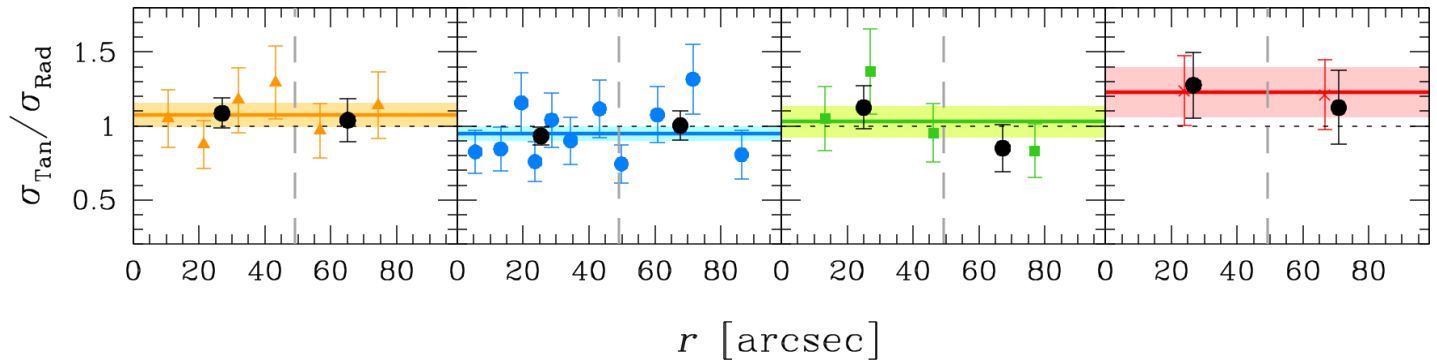

Figure 6. Tangential-to-radial anisotropy profile of the four populations on the RGBs of NGC 362 (defined as in Fig. 5). In each panel, points have the same colors and meaning as in Fig. 5. The horizontal lines represent the average over the entire FoV, the shaded regions correspond to $\pm 1 \sigma$ errors. The gray, dashed vertical line is set at $r_{\mathrm{h}}$. 
a chromosome map as we did for the RGB stars. Unlike the RGB investigation, we made use of a UV-filter-only map.

In panels (a1) and (a2) of Fig. 7, we illustrate the construction of the SGB chromosome map. First, we rectified the SGBs in the $m_{\mathrm{F} 814 \mathrm{~W}}$ versus $\left(m_{\mathrm{F} 336 \mathrm{~W}}-m_{\mathrm{F} 438 \mathrm{~W}}\right)$ and $m_{\mathrm{F} 814 \mathrm{~W}}$ versus $\left(m_{\mathrm{F} 275 \mathrm{~W}}-m_{\mathrm{F} 336 \mathrm{~W}}\right)$ CMDs (panels a1 and a2) using the same method as in the RGB analysis. The red crosses in these panels represent the red SGB previously identified.

Then, we built the $\Delta\left(m_{\mathrm{F} 275 \mathrm{~W}}-m_{\mathrm{F} 336 \mathrm{~W}}\right)$ versus $\Delta\left(m_{\mathrm{F} 336 \mathrm{~W}}-m_{\mathrm{F} 438 \mathrm{~W}}\right)$ chromosome map. The Hess diagram of this chromosome map is presented in panel (a3). For the sake of clarity, we excluded from the Hess diagram of the chromosome map the red-SGB stars. The remaining stars in the plot can be tentatively separated in a main group and a tail by means of the black line in panel (a3). Hereafter, we refer to the stars above and below the black line as azure- and yellow-SGB stars, respectively.

In panels (a1) and (a2), we plot with azure dots and yellow triangles the stars in each of the two groups identified in the more-populated SGB, respectively. These two groups of SGB stars present a color inversion between $m_{\mathrm{F} 814 \mathrm{~W}}$ versus $\left(m_{\mathrm{F} 336 \mathrm{~W}}-m_{\mathrm{F} 438 \mathrm{~W}}\right)$ and $m_{\mathrm{F} 814 \mathrm{~W}}$ versus $\left(m_{\mathrm{F} 275 \mathrm{~W}}-m_{\mathrm{F} 336 \mathrm{~W}}\right)$ CMDs (panels a1 and a2), similar to the behavior of the mPOPs along the RGB. Furthermore, the yellow triangles are in agreement with the $1 \mathrm{G}$ RGB stars, suggesting that they might be a $1 \mathrm{G}$ population. The azure dots appear to be consistent with the $2 \mathrm{G}$ RGB populations $\mathrm{B}$ and $\mathrm{C}$, thus implying that they might be a $2 \mathrm{G}$ population.

In Fig. 7 , we present $\sigma_{\mu}$ (panels b), $\sigma_{\text {Rad }}$ (panels c), and $\sigma_{\operatorname{Tan}}$ (panels c) of SGB stars as a function of the radial distance. The velocity dispersion in each bin in the plot is computed by considering 62,50 , and 30 stars for the azure, yellow, and red SGBs, respectively. At the $1 \sigma$ level, the three SGB populations present the same kinematics.

In Fig.8 we show the tangential-to-radial anisotropy profile of the SGB stars. All SGB populations are found to be isotropic with the exception of the red-SGB stars, for which the profile is significantly radially anisotropic at the $3 \sigma$ level for $r<r_{\mathrm{h}}$.

\subsubsection{Main sequence}

The analysis of the RGB and SGB stars is limited by small number statistics. In this section we focus our attention on the more plentiful MS stars.

The mPOP tagging was performed as for the SGB stars using a UV-filter chromosome map, which shows an elongated distribution of stars. From the Hess di- agram of the chromosome map (panel a3 of Fig. 9), we found that the MS stars can be split into a main group (hereafter "MS blue", in analogy with other studies of MS mPOPs) and a tail ("MS red"). These two groups of stars are clearly separated in the $m_{\mathrm{F} 814 \mathrm{~W}}$ versus $\left(m_{\mathrm{F} 336 \mathrm{~W}}-m_{\mathrm{F} 438 \mathrm{~W}}\right)$ and $m_{\mathrm{F} 814 \mathrm{~W}}$ versus $\left(m_{\mathrm{F} 275 \mathrm{~W}}-\right.$ $m_{\mathrm{F} 336 \mathrm{~W}}$ ) CMDs (panels a1 and a2), while in colors based in at least one optical filter such separation is less evident. We considered only well-measured stars $^{5}$ with $18.8<m_{\mathrm{F} 814 \mathrm{~W}}<20.5$ because in this magnitude interval the separation between MS blue and red in the Hess diagram is clearer.

Stars that are O-rich/Na-poor $(1 \mathrm{G})$ are bluer than those O-poor/Na-rich $(2 \mathrm{G})$ in $\left(m_{\mathrm{F} 336 \mathrm{~W}}-m_{\mathrm{F} 438 \mathrm{~W}}\right)$ color, but redder in $\left(m_{\mathrm{F} 275 \mathrm{~W}}-m_{\mathrm{F} 336 \mathrm{~W}}\right)$ color (Piotto et al. 2015). As such, MS-blue stars are likely $2 \mathrm{G}$ stars, while MS-red objects belong to the $1 \mathrm{G}$ population. These considerations are in agreement with the photometric and chemical tagging performed for the RGB stars in Sect. 3.2.1.

For $r<r_{\mathrm{h}}$, both MSs share the same velocitydispersion profile (panels b, c, and d in Fig. 9). For $r_{\mathrm{h}}<r<2 r_{\mathrm{h}}$, the MS-blue stars appear to be radially (at the $2.4 \sigma$ level) and tangentially (at the $2.7 \sigma$ level) colder than the MS-red stars. Finally, both MSs also have the same kinematic profiles at the $1 \sigma$ level for $r>2 r_{\mathrm{h}}$.

The tangential-to-radial anisotropy (Fig. 10) shows that the two groups are isotropic within our FoV.

\subsection{Energy equipartition}

From numerical simulations, Trenti \& van der Marel (2013) and Bianchini et al. (2016) showed that the energy-equipartition state $\left(\sigma_{\mu} \propto m^{-\eta}\right.$ with $\eta=0.5$ and $m$ the stellar mass) cannot be achieved, on account of the Spitzer instability. Trenti \& van der Marel (2013) showed that the stellar velocity dispersion reaches a maximum value of $\eta \sim 0.15$ in the core and then evolves, as in the other part of the cluster, to $\eta \sim 0.08$.

We investigated the status of energy equipartition of NGC 362 as follows. First, we divided the MS in 10 equally-populated bins of 2583 stars between $19<$ $m_{\mathrm{F} 606 \mathrm{~W}}<24$. We considered only well-measured stars (both astrometrically and photometrically) in F606W and F814W filters.

We computed the velocity dispersion $\sigma_{\mu}$ and the median magnitude of the stars in each bin. Median magnitudes were transformed into masses using a Darthmouth

\footnotetext{
${ }^{5}$ In addition to the photometric selections described in Appendix B.1, we discarded all stars with $\mid$ RADXS $\mid>0.025$ in F275W, F336W, F438W, F606W and F814W filters.
} 

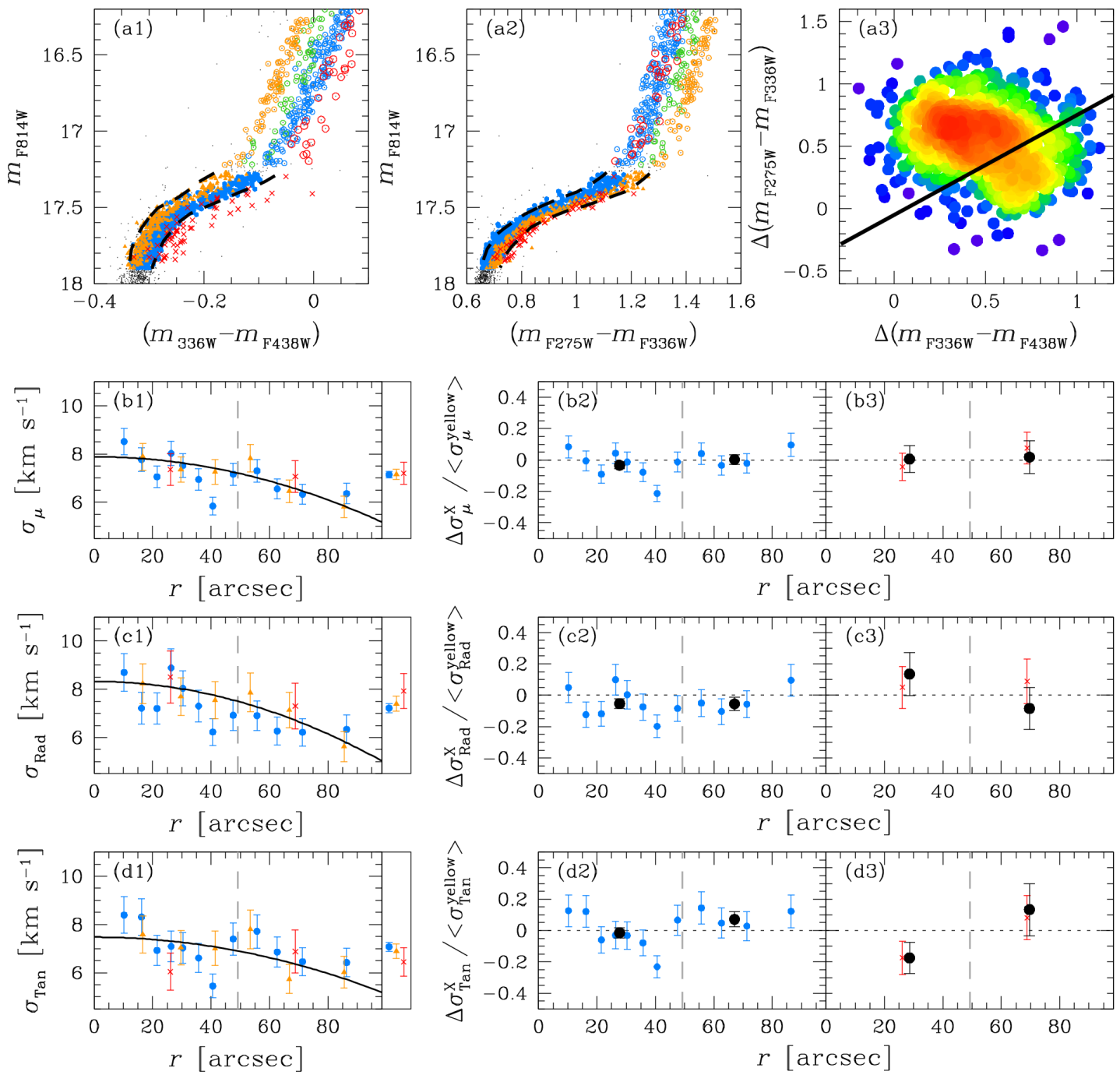

Figure 7. In panels (a1) and (a2) we present the $m_{\mathrm{F} 814 \mathrm{~W}}$ versus $\left(m_{\mathrm{F} 336 \mathrm{~W}}-m_{\mathrm{F} 438 \mathrm{~W}}\right)$ and the $m_{\mathrm{F} 814 \mathrm{~W}}$ versus $\left(m_{\mathrm{F} 275 \mathrm{~W}}-m_{\mathrm{F} 336 \mathrm{~W}}\right)$ CMDs of NGC 362, respectively. Open circles in panels (a1) and (a2) are RGB stars and are color-coded as in Fig. 5. The black, dashed lines are the fiducial lines adopted to rectify the SGBs analogous to the RGB study shown in Fig. 5. In panel (a3) we show the Hess diagram (in logarithmic scale) of the $\Delta\left(m_{\mathrm{F} 275 \mathrm{~W}}-m_{\mathrm{F} 336 \mathrm{~W}}\right)$ versus $\Delta\left(m_{\mathrm{F} 336 \mathrm{~W}}-m_{\mathrm{F} 438 \mathrm{~W}}\right)$ chromosome map of the SGB stars of NGC 362. We excluded from this map the red-SGB stars. Stars above this line are shown as azure dots in the previous panels, while the remaining stars are plot with yellow triangles. Red crosses represent red-SGB stars. Panels (b), (c), and (d) are similar to those in Fig. 5 but for the mPOPs hosted in the SGB. See the text for details.

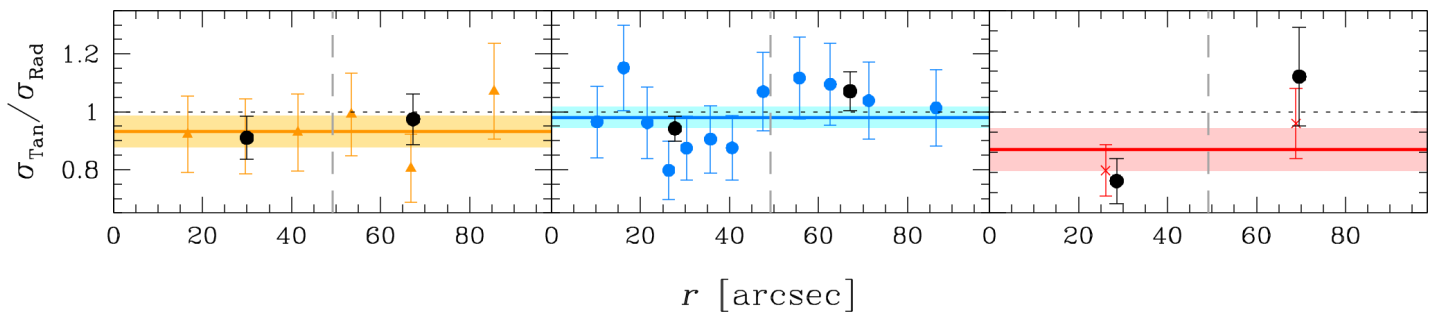

Figure 8. Similar to Fig. 6, but for the mPOPs on the SGB of NGC 362. 

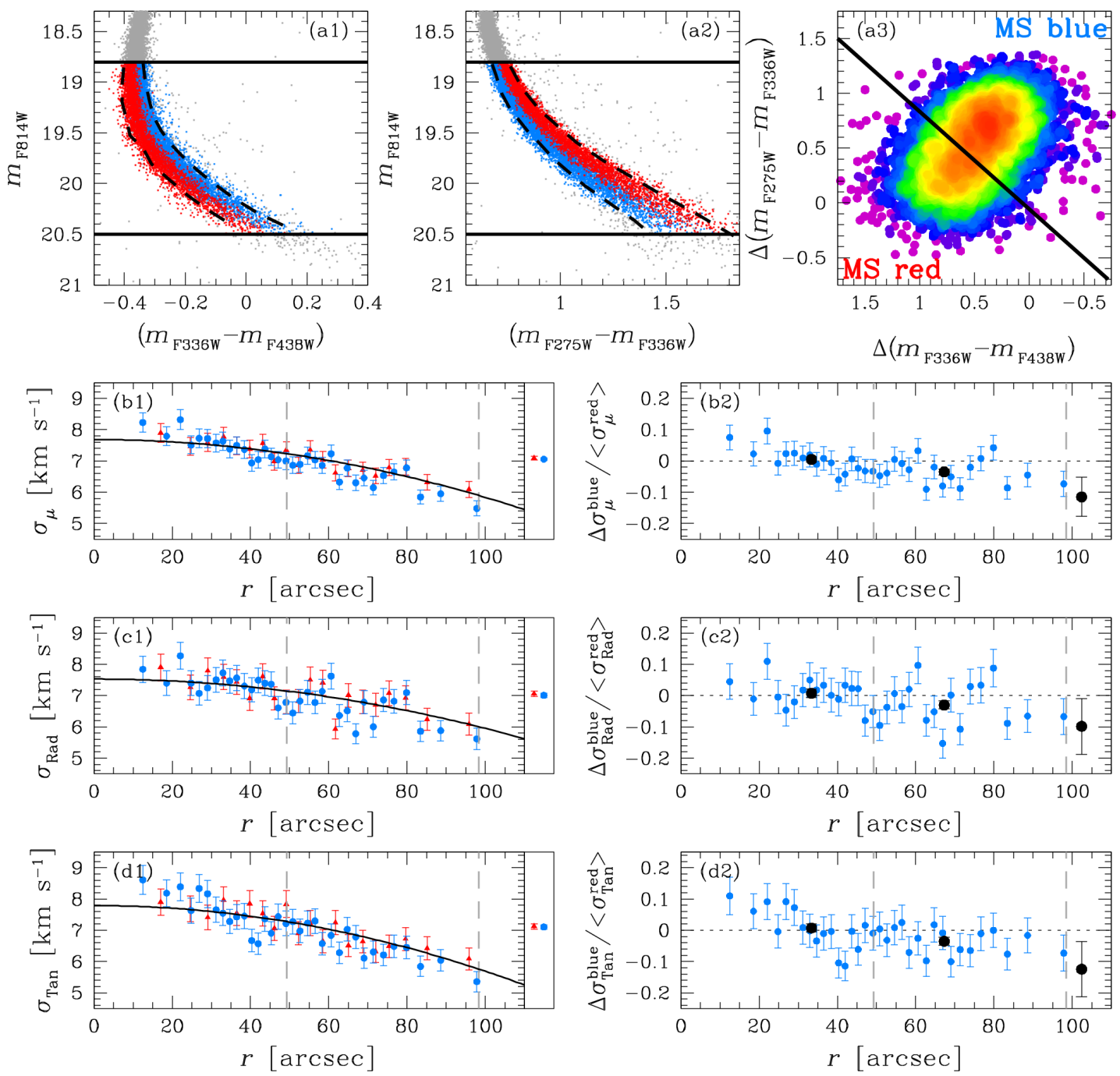

Figure 9. Similar to Fig. 7 but for MS stars. The velocity dispersion in each bin in of panels (b), (c), and (d) is computed by considering $180 \mathrm{MS}$ stars (except the outermost bin that is computed with 156 and 167 stars for MS red and blue, respectively). See the text for details.

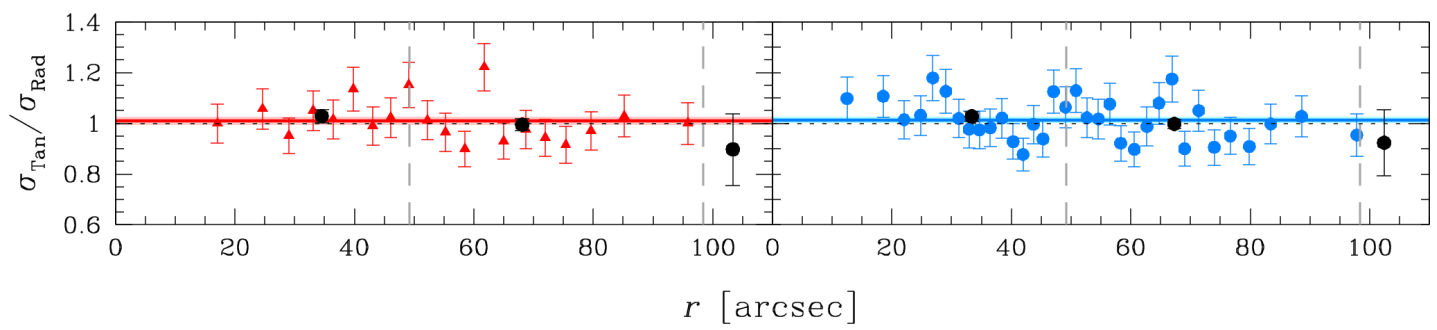

Figure 10. Similar to Figs. 6 and 8 but for MS stars. 
isochrone (Dotter et al. 2008) with $[\mathrm{Fe} / \mathrm{H}]=-1.26$, $\mathrm{E}(B-V)=0.06$, primordial He abundance and age of 11.46 Gyr (as in, e.g., Wagner-Kaiser et al. 2017). Finally, we fitted the $\sigma_{\mu}$ versus mass values in a loglog plane with a weighted least-squares straight line. The energy-equipartition parameter $\eta$ is the slope of this straight line. The result is summarized in panels (a) and (b) of Fig. 11. We find:

$$
\eta=0.114 \pm 0.012
$$

The half-mass relaxation time $t_{\mathrm{rh}}$ of a GC changes over time, either increasing or decreasing in response to the cluster's dynamical evolution, but its changes are expected to be small (Spitzer 1987). If we assume that the initial $t_{\mathrm{rh}}, t_{\mathrm{rh}}(0)$, is equal to $\sim 0.8 \mathrm{Gyr}$ as inferred

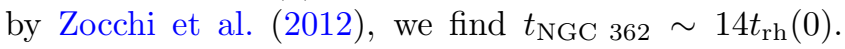
As such, our value of $\eta$ is in agreement with what is expected from Fig. 6 of Trenti \& van der Marel (2013).

Our estimate of the state of energy equipartition is obtained by considering all stars in the field, from the center to beyond $2 r_{\mathrm{h}}$. However, the level of energy equipartition in a GC is not the same at all distances. We also investigated the local level of energy equipartition in NGC 362 by dividing the sample into 5 radial bins of 25 arcsec each. The result is presented in panel (c).

The innermost interval presents $\eta \sim 0.4$, in contrast with the Trenti \& van der Marel (2013) $\eta_{\max }$ of $\sim 0.2$. However, our estimate of $\eta$ in the innermost radial bin was obtained by using stars covering a smaller mass range $\left(\Delta M \sim 0.2 M_{\odot}\right)$ than in all other bins in Fig. 11 $\left(\Delta M \sim 0.3 M_{\odot}\right)$, and might have been overestimated because of a poor straight-line fit.

The remaining points in panel (c) reveal that the level of energy equipartition decreases from $\sim 0.25$ to $\sim 0.08$ as the radial distance increases. This behavior is what we would expect as a result of the dynamical evolution of the cluster (Webb \& Vesperini 2017). The centermost regions of a $\mathrm{GC}$ are expected to be the first to relax.

As shown in panel (c), the global value of $\eta$ (black horizontal line) is not the average of the other points in the plot. The difference between $\sigma_{\mu}$ for a high-mass star at the center and at the outskirts of our field is smaller than for a low-mass star because of the combined effects of energy equipartition and hydrostatic equilibrium (see Fig. 4). As a consequence, we expect the local $\eta$ at the center to be higher than at the edge of the FoV because the low-mass stars at the center are kinematically hotter. Since the most of the faint stars are located in the outskirts of the FoV, the global value of $\eta$ is closer to the outermost than the innermost local values of $\eta$.

\subsubsection{To collapse or not collapse}

Previous studies of NGC 362 have not been able to conclusively determine whether this cluster in the postcore-collapsed phase. Chernoff \& Djorgovski (1989) and Trager et al. (1995) fit the surface brightness profile with a King model with concentration equal to 1.75 and 1.94, respectively, and classified NGC 362 as a possible postcore collapse cluster. Dalessandro et al. (2013) showed that the observed star-count profile of NGC 362 is well reproduced by either a mild power law $(\alpha \sim-0.2)$ or a double King profile (similar to what was found in the post-core-collapsed GC NGC 6752 by Ferraro et al. 2003), another indication that some dynamical processes have occurred in the cluster core. The advanced dynamical state of this cluster was also pointed out by Ferraro et al. (2012) from the analysis of the BS radial distribution in the context of the so-called "dynamical clock". Furthermore, the presence of two sequences of BSs in NGC 362 (see Sect. 3.4.1) and their radial distributions also seem to support the post-core-collapsed scenario (Ferraro et al. 2009; Dalessandro et al. 2013). However, none of these studies was able to clearly infer the pre- or post-core-collapsed state of NGC 362.

Recently, Bianchini et al. (2018) proposed the kinematic concentration $c_{k}$ as a diagnostic for core collapse, based entirely on the internal kinematics of a cluster. This parameter is defined as:

$$
c_{k}=\frac{m_{\mathrm{eq}}\left(r<r_{50}\right)}{m_{\mathrm{eq}}\left(r_{50}\right)}
$$

where $m_{\mathrm{eq}}\left(r<r_{50}\right)$ and $m_{\mathrm{eq}}\left(r_{50}\right)$ are the mass scale parameters measured by considering all stars within the $50 \%$ Lagrangian radius and between the $40 \%$ and $60 \%$ Lagrangian radii, respectively. The mass scale parameter $m_{\text {eq }}$ quantifies the level of energy equipartition of a cluster (Bianchini et al. 2016). In detail, Bianchini et al. (2016) describe the relation between the velocity dispersion $\sigma_{\mu}$ and the stellar mass as:

$$
\sigma(m)= \begin{cases}\sigma_{0} \exp \left(-\frac{1}{2} \frac{m}{m_{\mathrm{eq}}}\right) & \text { if } m \leq m_{\mathrm{eq}} \\ \sigma_{0} \exp \left(-\frac{1}{2}\right)\left(\frac{m}{m_{\mathrm{eq}}}\right)^{-\frac{1}{2}} & \text { if } m>m_{\mathrm{eq}}\end{cases}
$$

where $\sigma_{0}$ is the velocity dispersion for $m=0$. The relation between $\sigma_{\mu}$ and mass describes the different behavior of the energy equipartition for high- and low-mass stars. The cut-off at $m=m_{\text {eq }}$ avoids unphysical values of $\eta$. The relation between $\eta$ and $m_{\mathrm{eq}}$ is shown in Eq. 4 of Bianchini et al. (2016).

We estimated the local $\left(m_{\text {eq }}\left(r_{50}\right)\right)$ and global $\left(m_{\text {eq }}(r<\right.$ $\left.r_{50}\right)$ ) level of energy equipartition as follows. The velocity dispersions were computed as described in Sect. 3.3. Instead of the $50 \%$ Lagrangian radius, we adopted the 


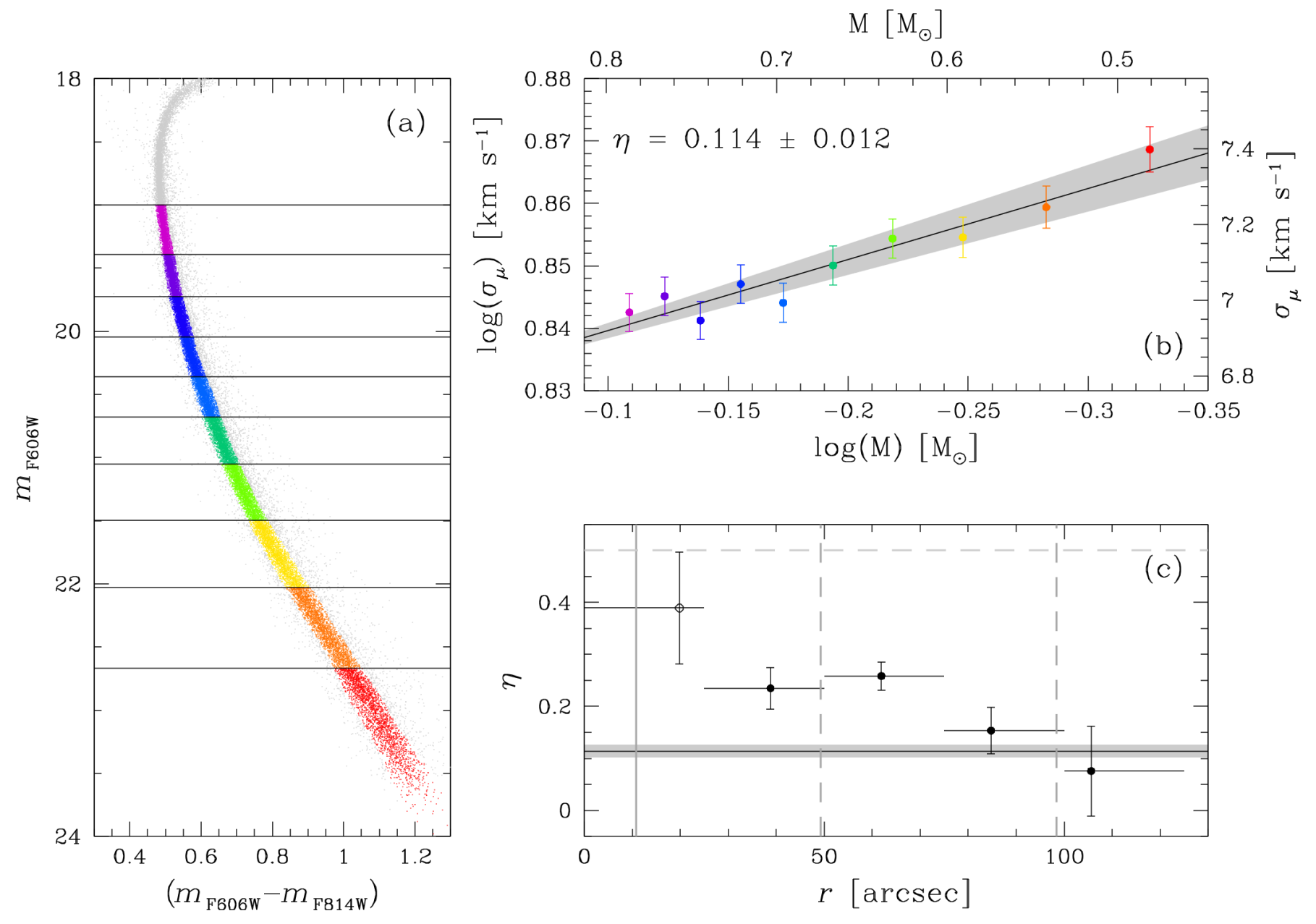

Figure 11. (a): $m_{\mathrm{F} 606 \mathrm{~W}}$ versus $\left(m_{\mathrm{F} 606 \mathrm{~W}}-m_{\mathrm{F} 814 \mathrm{~W}}\right)$ CMD of the MS of NGC 362 in which we defined 10 (equally-populated) bins color-coded from purple to red. Stars in these bins are used to compute the global level of equipartition of the cluster. The gray, horizontal lines define the limits of each magnitude bin. (b): combined velocity dispersion as a function of stellar mass. Both axes in the plot are in logarithmic scale. Points are color-coded as on the CMD in panel (a). The black line is the best fit straight line fit to the data. The slope of this line gives the level of energy equipartition $\eta$. (c): variation of $\eta$ as a function of radial distance. Each point is the local value of $\eta$ computed in steps of 25 arcsec from the center. Filled dots represent measurements in which stars adopted for the computation span a mass interval of $\sim 0.3 M_{\odot}$, while open circle indicates the case of $\Delta M \sim 0.2 M_{\odot}$. The horizontal error bars represent the radial interval of each point. The gray, solid vertical line is set at $r_{\mathrm{c}}$, while the two dashed lines are placed at $r_{\mathrm{h}}$ and $2 r_{\mathrm{h}}$, respectively. The gray, dashed horizontal line refers to $\eta=0.5$.

half-light radius as it is a direct observable. This assumption is justified since the results obtained with either the $40 \%, 50 \%$ or $60 \%$ Lagrangian radii are similar (Bianchini et al. 2018). For $m_{\mathrm{eq}}\left(r_{50}\right)$, we considered the stars within $\pm 0.5 r_{\mathrm{c}}$ from $r_{\mathrm{h}}$. Then, we performed a weighted least-square fit to the data using Eq. 3 with $\sigma_{0}$ and $m_{\text {eq }}$ as free parameters. We find:

$$
\left\{\begin{array}{l}
m_{\mathrm{eq}}\left(r<r_{50}\right)=1.60 \pm 0.32 M_{\odot} \\
m_{\mathrm{eq}}\left(r_{50}\right)=1.03 \pm 0.18 M_{\odot}
\end{array}\right.
$$

which for NGC 362 implies:

$$
c_{k}=1.54 \pm 0.41 .
$$

According to Bianchini et al. (2018), a cluster with $c_{k}>1$ has reached the core-collapsed state. Our result is the first inference of the post-core-collapsed state of NGC 362 based solely on kinematics. For the sake of completeness, we also computed the value of $c_{k}$ by adopting the half-mass radius given in Zocchi et al. (2012) and found $c_{k}=1.31 \pm 0.35$, further confirming NGC 362 to be a post-core-collapse GC.

The value of $m_{\mathrm{eq}}\left(r<r_{50}\right)$ is also qualitatively in agreement with the predictions of Fig. 6 of Bianchini et al. (2016), although their simulations did not involve core-collapse GCs. In particular, Bianchini et al. (2016) provide a relation between $m_{\mathrm{eq}}\left(r<r_{50}=r_{\mathrm{h}}\right)$ and $n_{\text {rel }}=t_{\text {age }} / t_{\mathrm{rc}}$, the ratio between the cluster age and the core relaxation time. For $t_{\text {age }}=11.46$ Gyr (Wagner- 
Kaiser et al. 2017) and $t_{\mathrm{rc}}=0.06$ Gyr (Harris 1996, 2010 edition), we obtain from their Eq. $6 m_{\mathrm{eq}}\left(r<r_{50}\right) \sim 1.60$, in excellent agreement with our estimate.

\subsection{Blue stragglers}

In Paper IV, we measured cluster kinematics to estimate the masses of BSs in a number of GCs. We repeat this analysis here using our updated catalog.

BSs are stars bluer and brighter than the MS turn-off, that is, they apparently sit on the MS where stars more massive than those at the turn-off would be found. It is thought that they are still present on the MS and have not evolved off as expected for stars of that mass because they gained their mass only recently, most likely as a result of stellar collisions or binary evolution (e.g., Knigge et al. 2009). As GCs are collisional systems, the stars interact and share energy. As a result, the clusters exhibit some degree of energy equipartition whereby more massive stars move more slowly than lower mass stars. If the BSs are indeed more massive than the RGB stars then it follows that we should expect the BSs to be moving more slowly than the RGB stars. It is this that allows us to use kinematics to estimate the mass of a BS population in a cluster. This approach is similar to that described by Parada et al. (2016), where they compared the radial distributions of BSs with those of other stellar populations to infer the mass of the BSs in 47 Tuc.

To do this, we estimate the velocity dispersion profiles of the BSs and the RGB stars from the kinematic data. First, we selected BSs from the $m_{\mathrm{F} 606 \mathrm{~W}}$ vs. $\left(m_{\mathrm{F} 606 \mathrm{~W}}-\right.$ $\left.m_{\mathrm{F} 814 \mathrm{~W}}\right) \mathrm{CMD}$ and computed their $\sigma_{\mu}$ as a function of radius in 5 equally-populated bins each containing 8 stars. The result is shown in Fig. 12. Blue dots represent the $\sigma_{\mu}$ values of BSs computed with our new PMs, the gray dots are from Paper IV. The two trends are in good agreement.

Then, we selected all well-measured NGC 362 members with $m_{\mathrm{F} 606 \mathrm{~W}}<19.8$ (i.e., one magnitude below the MS turn-off). We computed the combined velocitydispersion profile in (i) one bin with only stars within 2 arcsec from the center of NGC 362, (ii) five equallypopulated bins from 2 arcsec out to $r_{\mathrm{c}}$, (iii) 5 bins from $r_{\mathrm{c}}$ to $r_{\mathrm{h}}$, and (iv) 10 bins from $r_{\mathrm{h}}$ to the edge of the FoV.

We assume the velocity profiles of the BSs and the RGB stars have the same shape but that the BS dispersions are a fraction $\alpha$ lower owing to their mass difference, that is $\sigma_{\mathrm{BS}}=\alpha \sigma_{\mathrm{RGB}}$.

In our previous work, this was a two-step process. In Paper II, we measured the PM dispersion from NGC 362 from the catalog in Paper I and to this we fit a monotonically-decreasing $4^{\text {th }}$-order polynomial that was forced to be flat at the center. In Paper IV, we measured the dispersion profile for the BSs, used the best-fitting RGB polynomial as our fiducial profile, and estimated the factor $\alpha$ that provided the best fit to the BS profile. Here we are able to combine both steps together, and fit for the polynomial parameters and factor $\alpha$ simultaneously. We also fit the polynomial directly to the individual stars and not to the dispersion profile. We use the affine-invariant Markov-Chain Monte Carlo (MCMC) method EMCEE (Foreman-Mackey et al. 2013) to find and sample the region of parameter space that best fits the data. We extract 10000 points from the final MCMC parameter distributions (100 points at each of 100 steps, selected at 10-step intervals) to use as our fit sample.

Figure 13 shows the best-fitting polynomials, along with the dispersion profiles previously calculated. Note, the dispersion profiles are shown for visualization purposes, the fits were performed on individual stars. The black points show the measured dispersion profile for the RGB stars and the blue points show the measured dispersion profile for the BSs as in Fig. 12 .

For each point in our final fit sample, we calculate the predicted RGB and BS dispersion profiles. The gray and blue lines show the medians of those profiles for the RGB stars and BSs, respectively. The dark-gray and dark-blue areas show the region spanned by $1 \sigma$ (15.9 and 84.1) percentiles of the fitted profiles, and the lightgray and light-blue areas are show the region spanned by the $2 \sigma$ (2.3 and 97.7) percentiles of the fitted profiles. From these fits, we find $\alpha=0.81_{-0.07}^{+0.08}$, where the bestestimate is the median of the points that constitute our fit sample, and the uncertainties are estimated using the $1 \sigma$ percentiles.

In order to turn this velocity difference into a mass difference, we need to relate the scale factor $\alpha$ of the velocity dispersion profiles with scale factor $f$ of the masses, that is $M_{\mathrm{BS}}=f M_{\mathrm{RGB}}$. If we assume that the cluster is in partial equipartition then $\sigma \propto M^{-\eta}$, and it follows that $f=\alpha^{-\frac{1}{\eta}}$. In Paper IV, we had no estimate for $\eta$ directly, so we estimated $\eta$ from simulations by Bianchini et al. (2016) and assumed a boxcar distribution centered on $\eta$ and with half-width $\eta / 3$ in order to propagate our uncertainty in this value. We now have an estimate (and uncertainty) for $\eta$ at the average distance of the BSs $(r \sim 40 \operatorname{arcsec}): \eta=0.23 \pm 0.04$ (see the bottom-right panel of Fig. 11 in Sect. 3.3). We choose to use this value here, but otherwise adopt a similar procedure as before and draw $\eta$ from a boxcar centered on 0.23 and with half-width 0.04 . We use the fit sample to calculate a mass ratio $f=2.45_{-0.83}^{+1.24}$, again taking the median and $1 \sigma$ percentiles of the resulting distribution. 

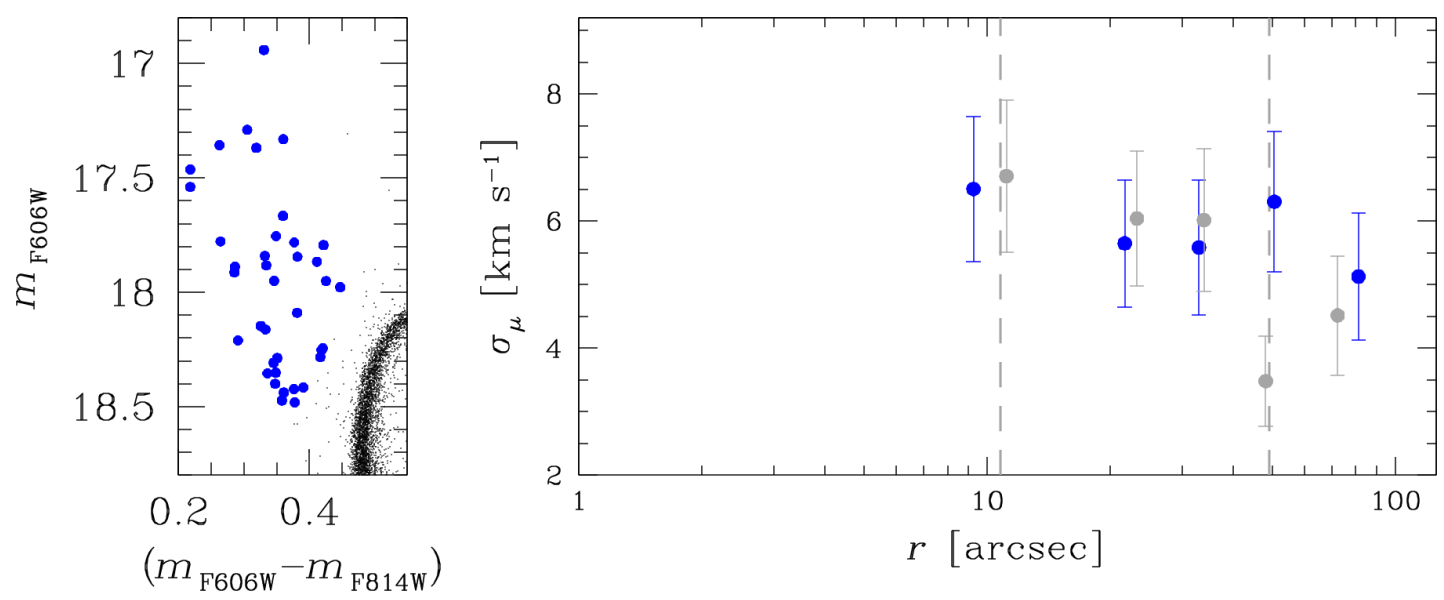

Figure 12. In the $m_{\mathrm{F} 606 \mathrm{~W}}$ versus $\left(m_{\mathrm{F} 606 \mathrm{~W}}-m_{\mathrm{F} 814 \mathrm{~W}}\right) \mathrm{CMD}$ on the left, we selected a sample of BSs (blue dots) that are used to compute the $\sigma_{\mu}$ radial profile on the right panel. Gray dots are the $\sigma_{\mu}$ values obtained in Paper IV using old PMs.

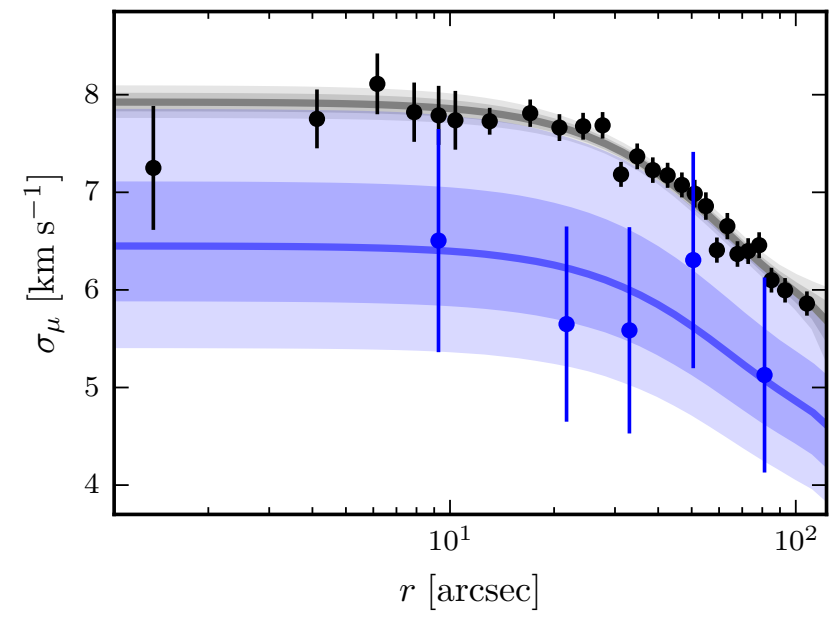

Figure 13. Measured velocity dispersion profiles for the RGB stars (black points) and the BSs (blue points). To the RGB stars, we fit a monotonically-decreasing $4^{\text {th }}$-order polynomial with a flat center; we assume that that BS profile is the same shape but rescaled by a factor $\alpha$. The black and blue lines show the median of the resulting fits, and the dark and light shaded regions show the $1 \sigma$ and $2 \sigma$ percentile regions.

Finally, we can estimate the mass of the BS stars. In Paper IV, we used isochrone fitting to estimate the mass of stars at the MS turn-off and assumed that the RGB stars have the same mass, thus $M_{\mathrm{RGB}}=0.80 M_{\odot}$. Combining this value with our estimate of mass fraction $f$, we obtain a final mass estimate for the BSs of $M_{\mathrm{BS}}=1.96_{-0.66}^{+0.99} M_{\odot}$. This is smaller than our previous estimate $2.18_{-0.42}^{+0.73} M_{\odot}$, but in agreement, within the uncertainties.

This value of the BS mass remains the largest mass estimate among those provided in Paper IV, and is somewhat offset from the mean value found for all the clusters of $\bar{M}_{\mathrm{BS}}=1.22 \pm 0.12 M_{\odot}$. A possible explanation of the discrepancy might be the incomplete sample of BSs analyzed, in particular close to the center of NGC 362. Dalessandro et al. (2013, see Fig. 9) found that the median distances of red- and blue-sequence BSs in NGC 362 are 15 and 28 arcsec, respectively. If we use the value of $\eta$ in Fig. 11 at these distances $(\eta=0.39 \pm 0.11)$ and assume that our BS sample is representative of all BSs in NGC 362 , the mass of the BS decreases to $1.36_{-0.30}^{+0.40} M_{\odot}$, in good agreement with the overall mean value found previously.

\subsubsection{The two BS populations of NGC 362}

NGC 362 is known to host two sequences of BSs (Dalessandro et al. 2013). The red sequence should be constituted by BSs resulting from mass-transfer binaries, the blue sequence from collisions. We studied the global kinematic behavior of these stars as follows.

We defined the two samples of BSs as shown in Fig. 7 of Dalessandro et al. (2013), i.e., by defining the fiducial loci of the two groups of BSs in the $m_{\mathrm{F} 555 \mathrm{~W}}$ versus $\left(m_{\mathrm{F} 555 \mathrm{~W}}-m_{\mathrm{F} 814 \mathrm{~W}}\right)$ CMD. The well-measured ${ }^{6} \mathrm{BSs}$ for the kinematic analysis are shown in the CMD in Fig. 14. For each of the two BS populations, we computed the value of $\sigma_{\mu}, \sigma_{\mathrm{Rad}}, \sigma_{\mathrm{Tan}}$, and the tangentialto-radial anisotropy in one radial bin covering the entire FoV (right panels in Fig. 14).

The red and blue BSs share the same kinematics at the $1 \sigma$ level. There is only a negligible difference in the tangential component $\sigma_{\text {Tan }}$ between red and blue BSs that goes in the direction that we would expect from their formation mechanisms.

\footnotetext{
6 The sample of well-measured BSs defined in Sect. 3.4 was refined by applying the photometric selections described in Appendix B.1 to the WFC3/UVIS filters F555W and F814W used for the BS tagging.
} 

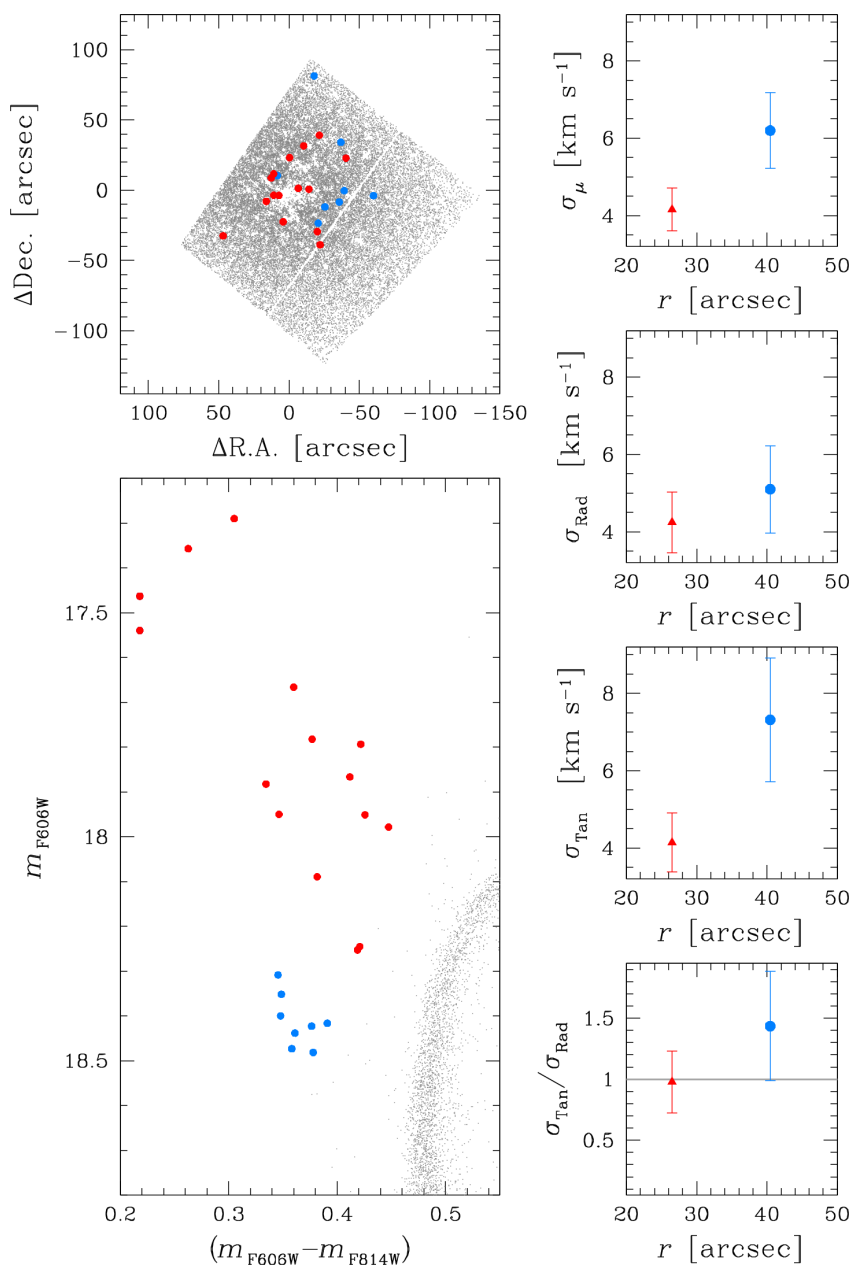

Figure 14. Kinematic analysis of the two BS groups in NGC 362 (as defined on the $m_{\mathrm{F} 606 \mathrm{~W}}$ versus $\left(m_{\mathrm{F} 606 \mathrm{~W}}-\right.$ $\left.m_{\mathrm{F} 814 \mathrm{~W}}\right)$ CMD of the bottom-left panel). We find the red BSs to be more centrally concentrated than the blue BSs (top-left panel), which was also reported by Dalessandro et al. (2013). In the right-hand panels, we show (from top to bottom) the combined velocity dispersion $\left(\sigma_{\mu}\right)$, the radialvelocity dispersion $\left(\sigma_{\text {Rad }}\right)$, the tangential-velocity dispersion $\left(\sigma_{\text {Tan }}\right)$ and the tangential-to-radial anisotropy radial profiles.

Finally, the red BSs are found to be more concentrated than the blue BSs, confirming the results of Dalessandro et al. (2013).

\subsection{Cluster rotation}

To date, only a handful of PM-based studies of the rotation of GCs in the plane of the sky have been performed (van Leeuwen et al. 2000; Anderson \& King 2003; Massari et al. 2013; Bellini et al. 2017d; Heyl et al. 2017; Bellini et al. 2018).

Regarding our target, Jeffreson et al. (2017) concluded from a spectroscopic analysis that the rotation of NGC 362 is small, if present at all. More recently, Kamann et al. (2018) found a maximum rotation of $\sim 2.3$ $\mathrm{km} \mathrm{s}^{-1}$ at the center, decreasing to $0.6 \mathrm{~km} \mathrm{~s}^{-1}$ at a distance of 1 arcmin. Here, we measured the amount of rotation of NGC 362 in three different ways.

Our PMs are computed by adopting the cluster stars as reference, and as such any direct trace of rotation in the reference population is canceled out by our linear transformations. However, any sign of rotation would be transferred (with the opposite sign) to background/foreground objects in the field.

NGC 362 is located in front of the SMC. As such, we used the SMC background stars to probe the rotation of NGC 362 in the plane of the sky as was done by Bellini et al. (2017d) for 47 Tuc. To exclude outliers from our data, we selected only stars with a PM error lower than 1 mas $\mathrm{yr}^{-1}$ and in which the PM fit was performed with at least 50 images, but we did not apply any other PMbased selection.

We selected the SMC stars in the VPD (top-left panel of Fig. 15), divided them in 6 equally-populated radial bins (85 stars per bin), and measured the tangential component of the PM $\mu_{\text {Tan }}$ in each bin. We find no evidence of plane-of-the-sky rotation for NGC 362 (bottomright panel of Fig. 15).

We also used an alternative method to infer the presence of rotation as was done by Massari et al. (2013). We fit a straight line to the tangential component of the PM of the SMC stars as a function of distance from the center of NGC 362. The slope of the straight line is consistent with 0 , meaning that the cluster is not rotating.

Finally, we used a third method, starting with the assumptions made by Heyl et al. (2017) in their PM analysis of the GC 47 Tuc. The authors found that the PMs of 47 Tuc stars are skewed in the tangential direction, implying that the cluster is rotating in the plane of the sky. The rotating nature of 47 Tuc had been previously inferred and characterized by Bellini et al. (2017d) with a multi-field analysis similar to that we used for NGC 362. This skew-based method is of particular interest when no other reference systems than the cluster itself are available. More recently, the same technique was adopted to infer the presence of differential rotation in the mPOPs of the GC $\omega$ Cen (Bellini et al. 2018).

Following Bellini et al. (2018), we computed the amount of skew in our PMs by measuring (i) the skewness value $G_{1}$ and the corresponding significance test $Z_{G_{1}}$ (Cramer 1997), and (ii) the third-order Gauss-Hermite moment $h_{3}$ (e.g., van der Marel \& Franx 1993). In general, a symmetric distribution has $-0.5<G_{1}<0.5$ (Bulmer 1979). The significance level of this value is given by $Z_{G_{1}}$ : if $\left|Z_{G_{1}}\right|>2$, the result is significant at a $>2 \sigma$ level, otherwise no conclusion can 

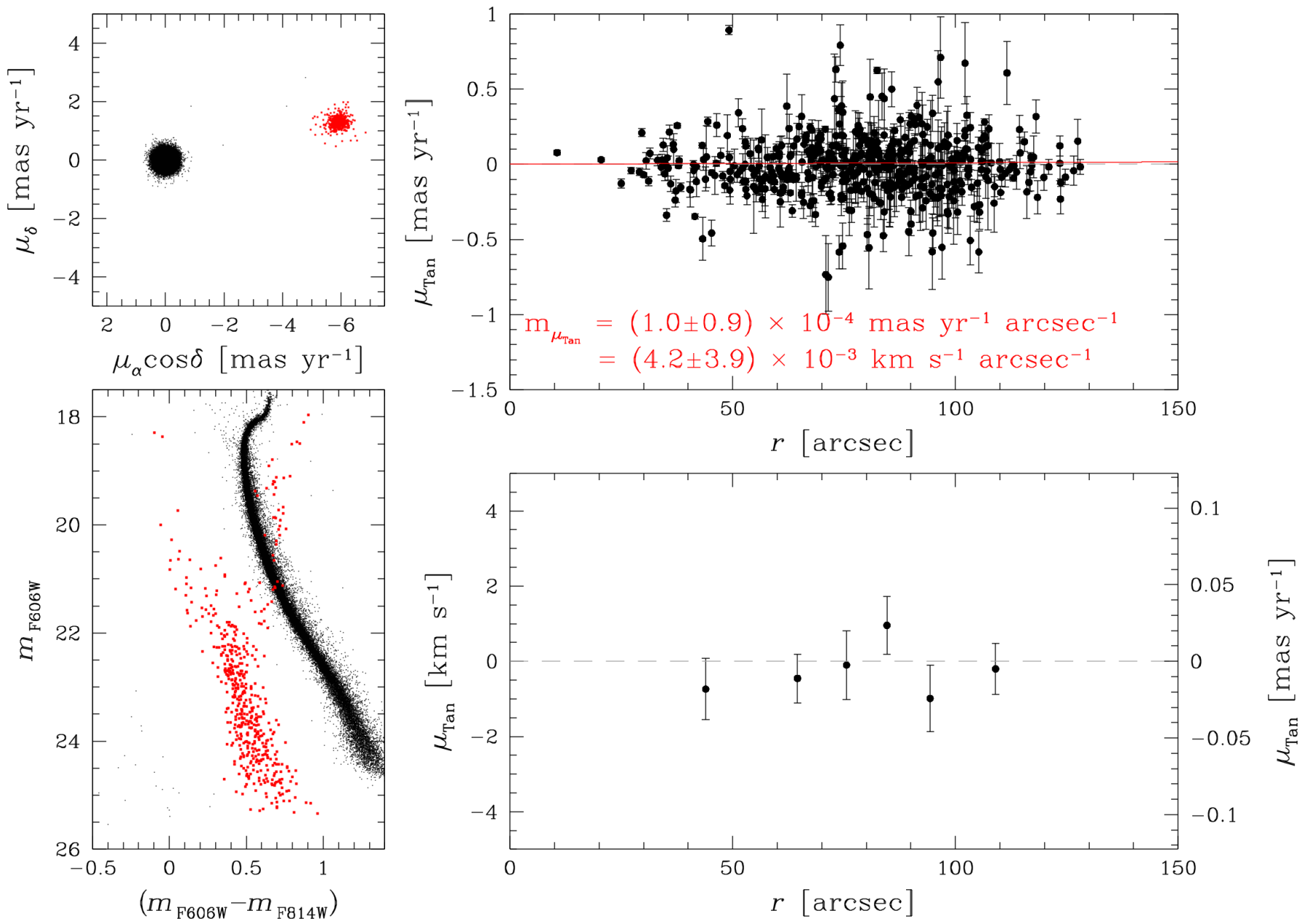

Figure 15. (Top-left): VPD of relative PMs. Members of NGC 362 are centered on the origin (black dots), while SMC stars have a distribution centered around $(-5.9,1.3) \mathrm{mas} \mathrm{yr}^{-1}$ (red points). (Bottom-left): $m_{\mathrm{F} 606 \mathrm{~W}}$ versus $\left(m_{\mathrm{F} 606 \mathrm{~W}}-m_{\mathrm{F} 814 \mathrm{~W}}\right) \mathrm{CMD}$ of NGC 362 (black) and SMC (red) stars. (Bottom-right): $\mu_{\text {Tan }}$ of SMC stars as a function of distance from the center of NGC 362. (Top-right): tangential component of the PMs $\left(\mu_{\mathrm{Tan}}\right)$ of SMC stars as a function of distance from the center. The gray, dashed horizontal line is set at 0 mas $\mathrm{yr}^{-1}$. The red line is a straight-line fit to the points. Both panels on the right indicate that NGC 362 is not rotating in the plane of the sky.

be inferred. The significance level of $h_{3}$ is instead given by its error.

For NGC 362 we find:

$$
\left\{\begin{array}{l}
G_{1}=-0.01 \\
Z_{G_{1}}=-0.71 \\
h_{3}=-0.006 \pm 0.004
\end{array} .\right.
$$

The values of $G_{1}$ and $Z_{G_{1}}$ suggest that the PM distribution is not skewed at the $3 \sigma$ level. The third-order Gauss-Hermite moment $h_{3}$ provides further support to the conclusion that NGC 362 has no internal rotation.

\subsection{Absolute PM}

We visually inspected the $H S T$ images of the cluster and identified two background galaxies, which we used to compute the absolute PM of NGC 362. The PMs of the two galaxies did not pass our astrometric qualityselection criteria (on account of their non-point-source nature). However, we are interested in high accuracy and not necessary in high precision to infer the absolute $\mathrm{PM}$ of the cluster.

Since our PMs are computed relative to the bulk motion of NGC 362, the background galaxies have a motion equal to the absolute PM of NGC 362, but with opposite sign. We find (see Fig. 16):

$$
\begin{aligned}
& \left(\mu_{\alpha} \cos \delta, \mu_{\delta}\right)_{\mathrm{NGC}} 362 \\
& =(6.703 \pm 0.278,-2.407 \pm 0.135) \text { mas } \mathrm{yr}^{-1} .
\end{aligned}
$$

The errors are the standard errors in the mean. It is straightforward to measure the absolute PM of SMC stars in our field. We refer to Appendix C for the analysis of the PMs of the SMC stars. 
The expected effect of parallax in our FoV given the temporal coverage of our images is lower than 0.026 mas $\mathrm{yr}^{-1}$ (see Appendix C). Therefore, we chose not to correct it because it is within the PM uncertainties of the reference galaxies.

As discussed in, e.g., Libralato et al. (2018), the absolute PM measured in a given field can be the combination of the amount of rotation of the system and of the motion of the center of mass (COM). Furthermore, projection effects arise when there are different lines of sight between the COM and the analyzed field. All these effects must be taken into account to infer the true absolute PM of NGC 362. However, in our case we do not have to include any of these contributions because (i) our field is centered on NGC 362, and (ii) we did not measure any systemic rotation (Sect. 3.5).

In the top-right panel of Fig. 16, we present the VPD of the absolute PM of stars in our field. The azure point represents our estimate of the absolute PM of NGC 362. In the bottom-right VPD we zoom-in around the location of our estimate of the absolute PM of NGC 362 and we compare it with literature values. We find an excellent agreement with the most-recent PM values given by Narloch et al. (2017) and with the value inferred with the PMs of the Gaia DR2 catalog by Gaia Collaboration et al. (2018b).

\section{CONCLUSIONS}

In this sixth paper of the series, we present an improved version of the data reduction aimed at better characterizing the PMs of stars in crowded environments, and applied it to the GC NGC 362.

Thanks to the new reduction pipeline we were able to (i) increase the number of detected sources and improve the overall PM astrometric precision by a factor of 3-5 with respect to the previous version of the PM catalog (Paper I), particularly in the innermost regions of the $\mathrm{GC}$, (ii) reach a $\mathrm{PM}$ precision of $\sim 10 \mu \mathrm{as} \mathrm{yr}^{-1}$ for bright stars, and (iii) measure the PMs of faint stars with high precision (e.g., a factor 20 better than the expected endof-mission precision of Gaia at its faint end $m_{\mathrm{F} 606 \mathrm{~W}} \sim$ 21.5).

We separated the mPOPs along the RGB, SGB and MS of the cluster and measured their velocity dispersions. All mPOPs show the same kinematics. We find only a marginal signature of $2 \mathrm{G}$ RGB stars of population $\mathrm{B}$ having a lower tangential velocity dispersion than $1 \mathrm{G}$ stars (at the $2.2 \sigma$ level). This evidence is similar to what has been recently found in other GCs and with numerical simulations (Richer et al. 2013; Bellini et al. 2015, 2018), but the difference between $\sigma_{\mathrm{Rad}}$ and $\sigma_{\text {Tan }}$
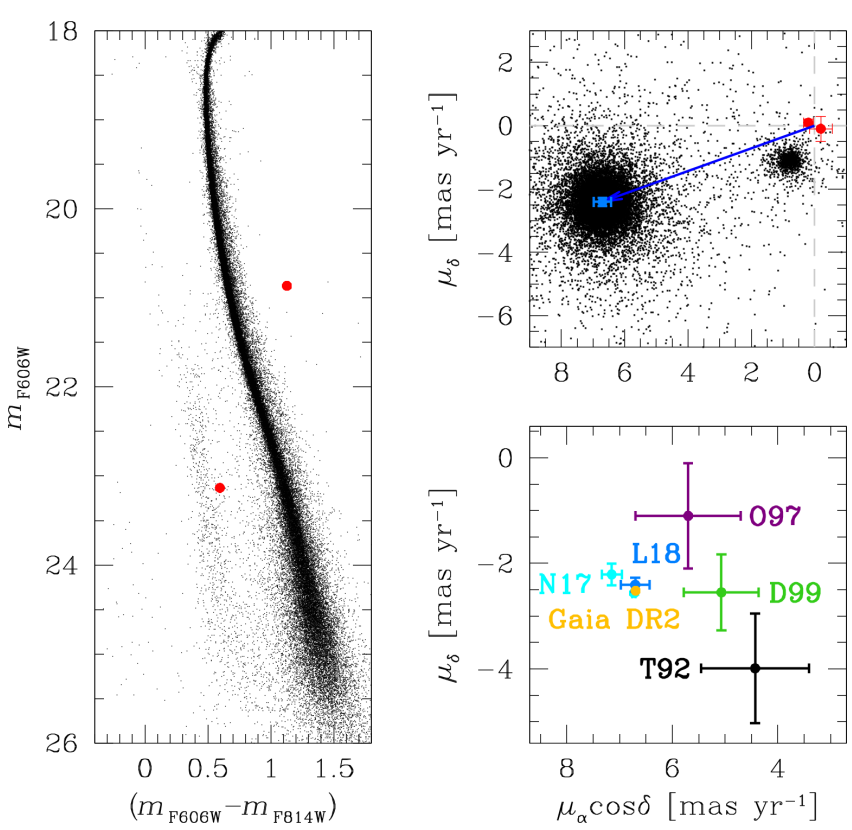

Figure 16. (Left): $m_{\mathrm{F} 606 \mathrm{~W}}$ versus $\left(m_{\mathrm{F} 606 \mathrm{~W}}-m_{\mathrm{F} 814 \mathrm{~W}}\right)$ CMD of NGC 362. The two galaxies used to compute the absolute PM of the cluster are shown as red dots. All other objects in the plot are depicted in black. (Top-right): VPD of the absolute PMs in equatorial coordinates. The red points (with error bars) are the PM of the galaxies. The blue line indicates the absolute motion of the cluster, the azure point with $1 \sigma$ uncertainty represents the absolute PM of NGC 362. (Bottom-right): Comparison of our estimate the absolute PM of NGC 362 (L18, azure) with the literature values: Tucholke (1992, T92, black), Odenkirchen et al. (1997, O97, purple), Dinescu et al. (1999, D99, green), and Narloch et al. (2017, N17, cyan). The estimate obtained with the PMs of the Gaia DR2 catalog is shown in yellow. The error bars of the Gaia-based PM have the same size of the point.

is not large enough to also create a significant radial anisotropy as in, e.g., NGC 2808 and $\omega$ Cen.

We also studied the level of energy equipartition of the cluster and its dependence on the radial distance from the cluster's center. Our results show that the degree of equipartition is stronger at smaller distances from the cluster's center in agreement with what expected from the effects of two-body relaxation (Trenti \& van der Marel 2013; Webb \& Vesperini 2017). Furthermore, we inferred that NGC 362 is in a post-core-collapsed state by comparing the local and global levels of energy equipartition. The classification of NGC 362 as a post-core-collapsed cluster is based entirely on the internal kinematics of the cluster; previous studies based the surface-brightness profile showed that this cluster could be modeled by a high-concentration King model, but the studies were unable to provide a firm conclusion concerning its pre- or post-core-collapsed state. Our result provides an additional example of the key role that 
the study of the internal kinematics can play in building a complete dynamical picture of GCs.

We refined the estimate of the average dynamical mass of the BSs hosted in this cluster, finding good agreement with the previous value of the BS mass published in Paper IV. NGC 362 is also known to host two sequences of BSs. We analyzed their velocity-dispersion radial profiles and find no differences in the kinematics.

We investigated the rotation in the plane of the sky of NGC 362 in three different ways. We find no evidence of significant plane-of-the-sky rotation. Although projection effects might contribute to hide the presence of rotation, this result suggests that this cluster is in an advanced stage of its evolution and has lost most its initial angular momentum (see, e.g., Einsel \& Spurzem 1999; Ernst et al. 2007; Tiongco et al. 2017, for numerical studies illustrating the gradual loss of rotation during a GC's evolution).

Finally, we used two galaxies in the background to measure the absolute PM of NGC 362. As a by product of our investigation, we also calculated the absolute PM of the SMC stars in our field.
The PM catalog of NGC 362 is made available to the community. The description of the catalog is provided in Appendix D.

\section{ACKNOWLEDGMENTS}

M.L. and A.B. acknowledge support from STScI grant GO 13297. M.L. acknowledges partial support by the Università degli Studi di Padova Progetto di Ateneo CPDA141214 "Towards understanding complex star formation in Galactic globular clusters". The authors thank the anonymous Referee for the thoughtful suggestions that improved the quality of the paper. Based on observations with the NASA/ESA HST, obtained at the Space Telescope Science Institute, which is operated by AURA, Inc., under NASA contract NAS 5-26555. This work has made use of data from the European Space Agency (ESA) mission Gaia (https://www.cosmos. esa.int/gaia), processed by the Gaia Data Processing and Analysis Consortium (DPAC, https://www. cosmos.esa.int/web/gaia/dpac/consortium). Funding for the DPAC has been provided by national institutions, in particular the institutions participating in the Gaia Multilateral Agreement.

\section{REFERENCES}

Anderson, J., \& King, I. R. 2003, AJ, 126, 772

Anderson, J., \& King, I. R. 2004, Instrument Science Report ACS 2004-15, 51 pages,

Anderson, J., \& King, I. R. 2006, Instrument Science

Report ACS 2006-01, 34 pages,

Anderson, J., Sarajedini, A., Bedin, L. R., et al. 2008, AJ, 135, 2055

Anderson, J., \& Bedin, L. R. 2010, PASP, 122, 1035

Baldwin, A. T., Watkins, L. L., van der Marel, R. P., et al. 2016, ApJ, 827, 12, Paper IV

Bedin, L. R., King, I. R., Anderson, J., et al. 2008, ApJ, 678, 1279-1291

Bellini, A., \& Bedin, L. R. 2009, PASP, 121, 141

Bellini, A., Anderson, J., \& Bedin, L. R. 2011, PASP, 123, 622

Bellini, A., Anderson, J., van der Marel, R. P., et al. 2014, ApJ, 797, 115, Paper I

Bellini, A., Vesperini, E., Piotto, G., et al. 2015, ApJL, 810, L13

Bellini, A., Anderson, J., Bedin, L. R., et al. 2017a, ApJ, 842,6

Bellini, A., Anderson, J., van der Marel, R. P., et al. 2017b, ApJ, 842, 7

Bellini, A., Milone, A. P., Anderson, J., et al. 2017c, ApJ, 844, 164
Bellini, A., Bianchini, P., Varri, A. L., et al. 2017d, ApJ, 844,167

Bellini, A., Libralato, M., Bedin, L. R., et al. 2018, ApJ, 853, 86

Bianchini, P., van de Ven, G., Norris, M. A., Schinnerer, E., \& Varri, A. L. 2016, MNRAS, 458, 3644

Bianchini, P., Webb, J. J., Sills, A., \& Vesperini, E. 2018, MNRAS,

Bohlin, R. C. 2016, AJ, 152, 60

Bulmer, M. G., 1979, "Principles of Statistics". Dover.

Carretta, E., Bragaglia, A., Gratton, R. G., et al. 2009, A\&A, 505, 117

Carretta, E., Bragaglia, A., Gratton, R. G., et al. 2013, A\&A, 557, A138

Chernoff, D. F., \& Djorgovski, S. 1989, ApJ, 339, 904

Cioni, M.-R. L., Bekki, K., Girardi, L., et al. 2016, A\&A, 586, A77

Costa, E., Méndez, R. A., Pedreros, M. H., et al. 2009, AJ, 137,4339

Costa, E., Méndez, R. A., Pedreros, M. H., et al. 2011, AJ, 141, 136

Cramer, D., 1997, "Basic Statistics for Social Research". Routledge.

Dalessandro, E., Ferraro, F. R., Massari, D., et al. 2013, ApJ, 778, 135 
Deustua, S. E., Mack, J., Bajaj, V., \& Khandrika, H. 2017, Space Telescope WFC Instrument Science Report,

Dinescu, D. I., Girard, T. M., \& van Altena, W. F. 1999, AJ, 117, 1792

Dobbie, P. D., Cole, A. A., Subramaniam, A., \& Keller, S. 2014, MNRAS, 442, 1663

Dotter, A., Chaboyer, B., Jevremović, D., et al. 2008, ApJS, 178, 89-101

Eichhorn, H., \& Jefferys, W. H. 1971, Publications of the Leander McCormick Observatory, 16, 267

Einsel, C., \& Spurzem, R. 1999, MNRAS, 302, 81

Ernst, A., Glaschke, P., Fiestas, J., Just, A., \& Spurzem, R. 2007, MNRAS, 377, 465

Ferraro, F. R., Possenti, A., Sabbi, E., et al. 2003, ApJ, 595,179

Ferraro, F. R., Beccari, G., Dalessandro, E., et al. 2009, Nature, 462, 1028

Ferraro, F. R., Lanzoni, B., Dalessandro, E., et al. 2012, Nature, 492, 393

Foreman-Mackey, D., Hogg, D. W., Lang, D., \& Goodman, J. 2013, PASP, 125, 306

Gaia Collaboration, Prusti, T., de Bruijne, J. H. J., et al. 2016a, A\&A, 595, A1

Gaia Collaboration, Brown, A. G. A., Vallenari, A., et al. 2016b, A\&A, 595, A2

Gaia Collaboration, Brown, A. G. A., Vallenari, A., et al. 2018a, arXiv:1804.09365

Gaia Collaboration, Helmi, A., van Leeuwen, F., et al. 2018b, arXiv:1804.09381

Goldsbury, R., Richer, H. B., Anderson, J., et al. 2010, AJ, 140, 1830-1837

Harris, W. E. 1996, AJ, 112, 1487

Heyl, J., Caiazzo, I., Richer, H., et al. 2017, ApJ, 850, 186

Jeffreson, S. M. R., Sanders, J. L., Evans, N. W., et al. 2017, MNRAS, 469, 4740

Kallivayalil, N., van der Marel, R. P., Besla, G., Anderson, J., \& Alcock, C. 2013, ApJ, 764, 161

Kamann, S., Husser, T.-O., Dreizler, S., et al. 2018, MNRAS, 473, 5591

King, I. R. 1966, AJ, 71, 64

Knigge, C., Leigh, N., \& Sills, A. 2009, Nature, 457, 288

Libralato, M., Bellini, A., Bedin, L. R., et al. 2018, ApJ, 854,45

Lim, D., Lee, Y.-W., Pasquato, M., Han, S.-I., \& Roh, D.-G. 2016, ApJ, 832, 99

Massari, D., Bellini, A., Ferraro, F. R., et al. 2013, ApJ, 779,81

Milone, A. P., Piotto, G., Bedin, L. R., et al. 2012, A\&A, 540, A16

Milone, A. P., Piotto, G., Renzini, A., et al. 2017, MNRAS, 464, 3636
Narloch, W., Kaluzny, J., Poleski, R., et al. 2017, MNRAS, 471,1446

Niederhofer, F., Cioni, M.-R. L., Rubele, S., et al. 2018, arXiv:1801.07738

Odenkirchen, M., Brosche, P., Geffert, M., \& Tucholke, H.-J. 1997, NewA, 2, 477

Pancino, E., Bellazzini, M., Giuffrida, G., \& Marinoni, S. 2017, MNRAS, 467, 412

Parada, J., Richer, H., Heyl, J., Kalirai, J., \& Goldsbury, R. 2016, ApJ, 830, 139

Piatek, S., Pryor, C., \& Olszewski, E. W. 2008, AJ, 135, 1024

Piotto, G., Milone, A. P., Anderson, J., et al. 2012, ApJ, 760,39

Piotto, G., Milone, A. P., Bedin, L. R., et al. 2015, AJ, 149, 91

Richer, H. B., Heyl, J., Anderson, J., et al. 2013, ApJL, $771, \mathrm{~L} 15$

Spitzer, L. 1987, Princeton, NJ, Princeton University Press, 1987, 191 p.

Tiongco, M. A., Vesperini, E., \& Varri, A. L. 2017, MNRAS, 469, 683

Trager, S. C., King, I. R., \& Djorgovski, S. 1995, AJ, 109, 218

Trenti, M., \& van der Marel, R. 2013, MNRAS, 435, 3272

Tucholke, H.-J. 1992, A\&AS, 93, 293

van der Marel, R. P., \& Franx, M. 1993, ApJ, 407, 525

van der Marel R. P., Alves D. R., Hardy E., Suntzeff N. B., 2002, AJ, 124, 2639

van der Marel, R. P., \& Guhathakurta, P. 2008, ApJ, 678, 187-199

van der Marel, R. P., \& Anderson, J. 2010, ApJ, 710, 1063 van der Marel, R. P., \& Sahlmann, J. 2016, ApJL, 832, L23 van Leeuwen, F., Le Poole, R. S., Reijns, R. A., Freeman, K. C., \& de Zeeuw, P. T. 2000, A\&A, 360, 472

Vieira, K., Girard, T. M., van Altena, W. F., et al. 2010, AJ, 140, 1934

Wagner-Kaiser, R., Sarajedini, A., von Hippel, T., et al. 2017, MNRAS, 468, 1038

Watkins, L. L., van der Marel, R. P., Bellini, A., \& Anderson, J. 2015, ApJ, 803, 29, Paper II

Watkins, L. L., van der Marel, R. P., Bellini, A., \& Anderson, J. 2015, ApJ, 812, 149, Paper III

Webb, J. J., \& Vesperini, E. 2017, MNRAS, 464, 1977

Zocchi, A., Bertin, G., \& Varri, A. L. 2012, A\&A, 539, A65 


\section{APPENDIX}

\section{A. FIRST- AND SECOND-PASS PHOTOMETRY}

For the data reduction, we employed only fltt-type exposures, since they preserve the unresampled pixel data for appropriate point-spread-function (PSF) fitting. ACS/WFC and WFC3/UVIS _flc images have been pipeline corrected for charge-transfer-efficiency (CTE) defects as described in Anderson \& Bedin (2010). No CTE correction is available for ACS/HRC data ${ }^{7}$.

The first-pass photometry is performed with a single wave of finding, and no neighbor subtraction is applied prior to the PSF fit. We extracted positions and fluxes of all detectable sources via empirical PSF fitting. The PSF models of each exposure were obtained by perturbing the publicly-available ${ }^{8}$ library PSFs of each $H S T$ camera/filter. We derived spatially-variable perturbation PSFs for the ACS/WFC and WFC3/UVIS images, while a single (spatially-constant) perturbation PSF model was applied to ACS/HRC data. Stellar positions were corrected for geometric distortion by using the distortion solutions provided by Anderson \& King (2004, 2006), Bellini \& Bedin (2009) and Bellini et al. (2011).

Because it does no neighbor subtraction, the first-pass photometry is severely limited in the centermost regions of the cluster, where there is significant crowding. Our second-pass photometry is specifically designed to address this issue.

Before running the second-pass photometry algorithm, we need to set-up a common reference-frame system in which all stars can be measured consistently. We adopted a reference system in which the $X$ and $Y$ axes point toward West and North, respectively, and the center of the cluster (provided by Goldsbury et al. 2010) is placed at position $(5000,5000)$. The pixel scale of the master frame is set to be exactly 40 mas pixel ${ }^{-1}$, very similar to that of WFC3/UVIS, and an intermediate value between those of ACS/HRC and ACS/WFC. We set up orientation and scale of our reference system with the Gaia DR1 catalog. Then, we used only bright, unsaturated, relatively isolated stars in common between our single-exposure catalogs and the Gaia DR1 to derive general, six-parameter linear transformations that were used to iteratively cross-identify stars of each individual exposure on the master-frame plane. Master-frame positions are then the average of these transformed

\footnotetext{
${ }^{7}$ We investigated the presence of CTE-related systematic effects in our PMs and found them to be negligible.

8 http://www.stsci.edu/ jayander/STDPSFs/.
}

positions. For each filter, stellar magnitudes of each exposure were zero-pointed to match those of the longest available exposure before averaging.

The second-pass photometry is run using KS2, a sophisticated FORTRAN routine based on the code developed to reduce the "ACS Globular Cluster Treasury Survey" data (Anderson et al. 2008). KS2 starts from the outputs of the first-pass photometry (PSFs and transformations) and simultaneously reduces all individual epochs/filters/exposures at once. Objects are measured in three different methods by KS2. In this work we considered only the method-\#1 measurements, in which stellar positions and fluxes are obtained through PSF fitting of the individual neighbor-subtracted exposures. This method is best suited to high-precision PM analysis.

KS2 allows us to select a subset of exposures to be used for the finding stage. For this task we chose images taken with ACS/HRC (F435W) and ACS/WFC $(\mathrm{F} 606 \mathrm{~W}+\mathrm{F} 814 \mathrm{~W})$ for the following reasons. First, ACS/HRC data are the most suitable to probe the very center of this core-collapse GC (within its core radius $r_{\mathrm{c}}=0.18 \mathrm{arcmin}$ ), since they provide the highest angular resolution. Also, the GO-10775 ACS/WFC data were taken at a somewhat intermediate epoch and offer the largest overlap with other exposures. KS2 measures stars in single exposures if they are within a 1-pixel searching radius from the position measured during the finding stage. Some background objects, in particular SMC stars, have moved by more than 1 pixel between the first and the last available epoch, so choosing an intermediate epoch for the finding process allowed us to find and measure all sources.

KS2 outputs several diagnostic parameters: QFIT value $^{9}$, the magnitude rms, the fractional flux due to neighbors within the fitting radius prior to neighbor subtraction $(o)$, the ratio between the number of individual exposures used to measure a stellar position and flux and the total number of exposures actually found for the star $\left(r_{\mathrm{N}}\right)$, and the shape parameter RADXS ${ }^{10}$. These quality parameters will be used later in Sect. 3 to select the best-measured sources.

Finally, the KS2-based magnitudes were calibrated in the Vega-mag system following prescriptions given in

\footnotetext{
9 The QFIT represents the linear-correlation coefficient (similar to the Pearson coefficient) between the values of the real pixels and those of the PSF models. The closer to unity the QFIT is, the better the PSF fit.

10 The RADXS value is the excess/deficiency of flux outside of the fitting radius with respect to that predicted by the PSF. It is particularly effective is separating faint stars from artifacts and background galaxies (see also Bedin et al. 2008).
} 
Bellini et al. (2017a). Our photometry is corrected for differential reddening as described in Milone et al. (2012) and Bellini et al. (2017b). We refer to Bellini et al. (2017b) for the detailed description of the methodology.

\section{B. RELATIVE PROPER MOTIONS}

As a by product, KS2 also provides neighborsubtracted stellar positions and fluxes in the raw reference system of each exposure (hereafter, the raw catalogs): a clear advantage over the catalogs produced by the first-pass photometry. PMs are computed using these catalogs. We considered only stars measured in at least both F606W and F814W filters ${ }^{11}$, and we excluded all catalogs based on the F275W filter, because of color-dependent systematic effects in the $\mathrm{F} 275 \mathrm{~W}$ geometric-distortion correction (Bellini et al. 2011).

Paper I developed iterative procedures to compute high-precision stellar PMs. These procedures have been recently improved and discussed by Bellini et al. (2018), and represent the state-of-the-art in observations with $H S T$. In the following, we describe the outline of the PM computation and highlight the few but significant changes we applied to our data set.

Each iteration starts by cross-identifying stars in each raw catalog with those measured by KS2 on the master frame, PM-shifted at the epoch of the raw catalog, by means of general, six-parameter linear transformations of a set of reference bright, unsaturated cluster members. At the first iteration, stellar PMs are assumed to be zero, and membership is determined solely on the basis of stellar locations on the CMD. Since cluster members define the transformations, the computed PMs will be relative to the bulk motion of the cluster.

For a given star, its master-frame transformed positions as a function of epoch are fit with a least-squares straight line, the slope of which is a direct estimate of the star's PM. This fitting procedure is itself iterated, and involves data rejection and sigma clipping. We refer the reader to Paper I for more details. The last least-squares fit is performed with locally-transformed master-frame stellar positions, based on the closest 45 reference stars, as was done in Bellini et al. (2018). Local transformations help in mitigating large-scale systematic residuals.

At the end of each iteration, master-frame positions can be adjusted to match the epoch of each observation to minimize mismatches during the cross-identification step, as described in Bellini et al. (2018). The PM-

\footnotetext{
11 We have exposures in F814W filter obtained with either the ACS/WFC or the WFC3/UVIS cameras. In the paper, we always refer to the ACS/WFC F814W filter as simply F814W filter unless explicitly declared otherwise.
}

computation converges when there are at most negligible differences between master-frame positions from one iteration to the next.

\section{B.1. Correction of the PM systematics}

Bellini et al. (2018) computed the PMs of $\omega$ Cen using the same techniques described in this paper. In their analysis, they pointed out that the PMs were affected by low- and high-frequency systematic effects. The former are related to the different overlaps between the data sets, and hence with the temporal baseline. The latter are fine-scale structures due to uncorrected CTE and distortion residuals.

First, we considered only stars with (i) QFIT larger than the $85^{\text {th }}$ percentile at any given magnitude ${ }^{12}$, (ii) magnitude rms lower than the $85^{\text {th }}$ percentile, again at any given magnitude, (iii) flux greater than 2.5 times the local rms of the sky, (iv) o parameter smaller than 1, and (v) $r_{\mathrm{N}}$ ratio greater than 0.5 . We also excluded all stars fainter than $m_{\mathrm{F} 606 \mathrm{~W}} \sim 25.3$ (F606W instrumental magnitude $\sim-6.5)$. Finally, bonafide cluster members are defined as well-measured stars with a PM smaller than 1.2 mas $\mathrm{yr}^{-1}$ (panel a in Fig. 17) and lying within the two ridge lines (drawn by hand) in the $m_{\mathrm{F} 606 \mathrm{~W}}$ versus $\left(m_{\mathrm{F} 606 \mathrm{~W}}-m_{\mathrm{F} 814 \mathrm{~W}}\right)$ CMD of panel (b).

Low-frequency systematic effects reveal themselves as shifts of the bulk motion of cluster stars from the origin of the VPD. To correct for these low-frequency systematic effects, we first divided selected stars into different groups according to the temporal baseline used to compute their PMs. For each group, we then computed the $3 \sigma$-clipped median PM and corrected the PM of the stars accordingly.

The maps of the local PM components along $\alpha \cos \delta$ and $\delta$ (panels $\mathrm{c}$ and $\mathrm{d}$ ) reveal the presence of uncorrected high-frequency systematic effects with amplitude as large as \pm 0.2 mas $\mathrm{yr}^{-1}$. Each point in the maps is color-coded according to the median PM of the closest 100 reference stars. In order to remove these effects, we performed an a-posteriori correction as described in Paper I. Briefly, for each object we selected the closest 100 cluster stars. These stars were used to compute the high-frequency correction defined as the median value of their PM in each coordinate. In panels (e) and (f) we show the local PM maps after the high-frequency effects were corrected.

\footnotetext{
12 All objects with a QFIT larger than 0.99 are also included. Sources with a QFIT smaller than 0.6 are always discarded. A similar procedure was applied to the magnitude rms: stars with rms smaller than $0.01 \mathrm{mag}$ are always retained, and all objects with a magnitude rms larger than 0.25 mag are rejected.
} 

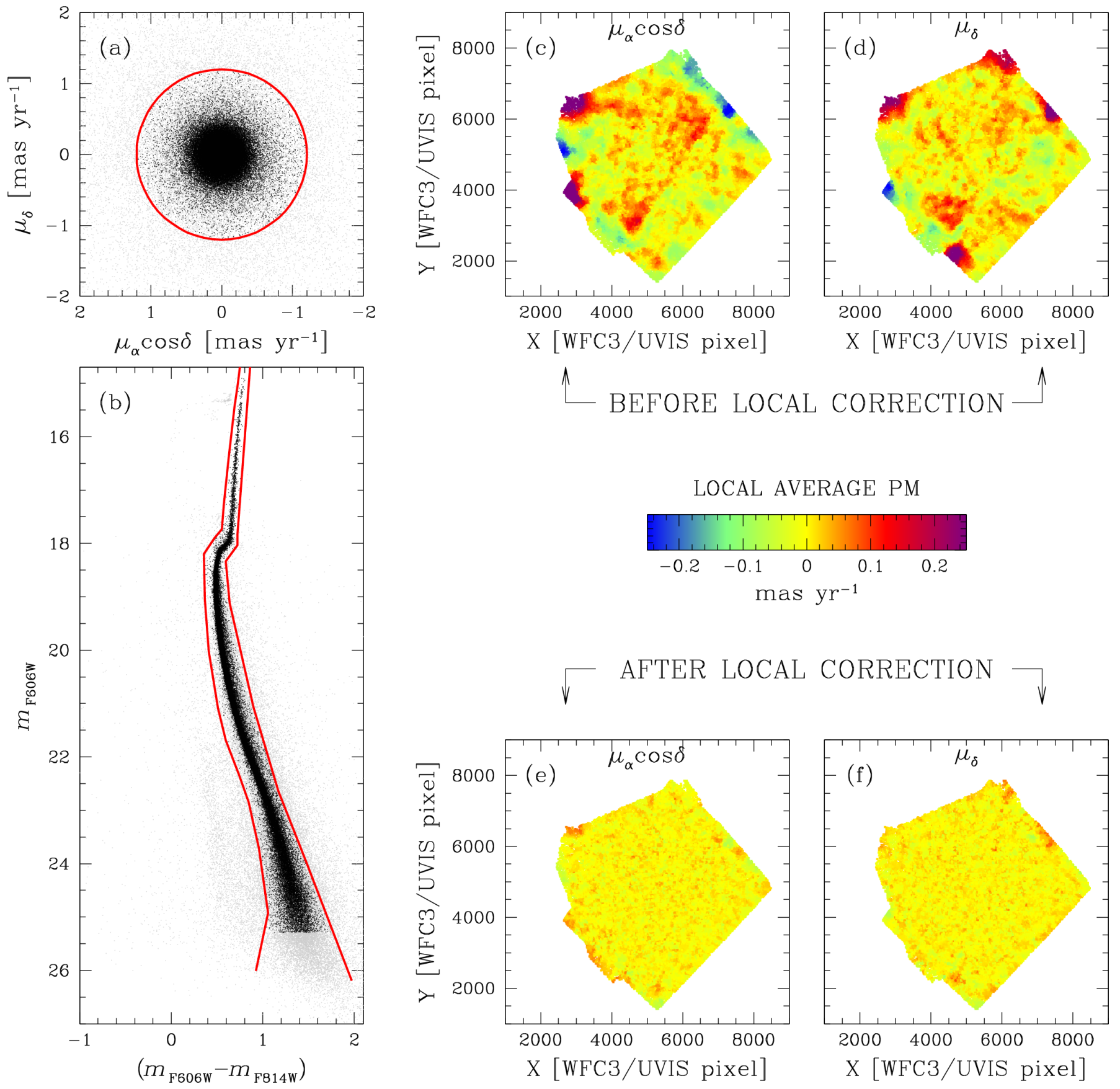

Figure 17. Overview of the correction for the high-frequency PM systematic effects. Well-measured cluster members are selected according to their PM in the VPD (within the red circle in panel a) and their location on the $m_{\mathrm{F} 606 \mathrm{~W}}$ versus $\left(m_{\mathrm{F} 606 \mathrm{~W}}-m_{\mathrm{F} 814 \mathrm{~W}}\right)$ CMD (within the two red lines in panel b). In panels (c) and (d) we show the maps of the local PM along $\alpha \cos \delta$ and $\delta$ (panel d) before the high-frequency correction. Each point is color-coded according to the color bar in the middle. The corresponding maps based on corrected PM are shown in panels (e) and (f) for $\mu_{\alpha} \cos \delta$ and $\mu_{\delta}$, respectively. 
As discussed in Bellini et al. (2018), at each step of the correction we included in the error budget the standard error on the median of the correction by summing it in quadrature with the PM errors, thus artificially increasing the intrinsic velocity dispersion of the cluster stars. This is not a problem for our studies of the internal kinematics of NGC 362 because we account for the PM uncertainties when we measure the observed PM dispersion. In any case, our PM catalog contains both the original (uncorrected) and the corrected PMs with the corresponding errors to allow users to choose the best option for their investigation.

\section{B.2. Comparison with the PM catalog of Paper I}

To better highlight the differences with the previous data reduction, we made a comparison between the old and new PMs. The result is presented in Fig 18.

In our analysis we considered all objects with a PM measurement and, for a fair comparison, we compared only the uncorrected PMs, since the two papers performed different PM corrections. In the top panel, we show a histogram of the objects in the catalog as a function of the distance from the center of NGC 362 . The new catalog has an overall higher number of objects compared to that of Paper I, in particular in the innermost 10 arcsec.

The astrometric precision reached in Paper I and in our paper for well-measured stars brighter than the SGB level is of about $30 \mu \mathrm{as} \mathrm{yr}^{-1}$. For stars at $m_{\mathrm{F} 606 \mathrm{~W}} \sim 18$, the PM precision achieved in our new catalog is typically three times better than that of Paper I. At the faint end of the Paper I catalog $\left(m_{\mathrm{F} 606 \mathrm{~W}} \sim 25\right)$, the median $\operatorname{err}_{\mu}$ value is $\sim 1.6$ mas $\mathrm{yr}^{-1}$, while in our new catalog the PMs at this magnitude level have a median error of $\sim 0.3$ mas $\mathrm{yr}^{-1}$, a factor five better.

Second-pass photometry is more effective than firstpass photometry in very crowded regions as the cluster's center because of the neighbor-subtraction stage. As such, we compared the PM errors of stars within 10 arcsec from the center of NGC 362 (second panel from the bottom in Fig. 18). The astrometric precision reached for bright stars at the SGB level very close to the center of NGC 362 is of the order of $10 \mu \mathrm{as} \mathrm{yr}^{-1}$ in our new catalog, and of $\sim 40 \mu \mathrm{as} \mathrm{yr}^{-1}$ in the old catalog, a factor 4 worse. For stars at the HB level $\left(m_{\mathrm{F} 606 \mathrm{~W}} \sim 15.3\right)$, the value of $\operatorname{err}_{\mu}$ is comparable.

In the bottom panel of Fig. 18 we compared only stars measured by using between 50 and 82 images (the maximum number of overlapping exposures of Paper I). The new PM errors in our paper are again a factor $\sim 4$ better than those in the old catalog, and the PM-error distribution looks overall tighter than that of the old catalog,
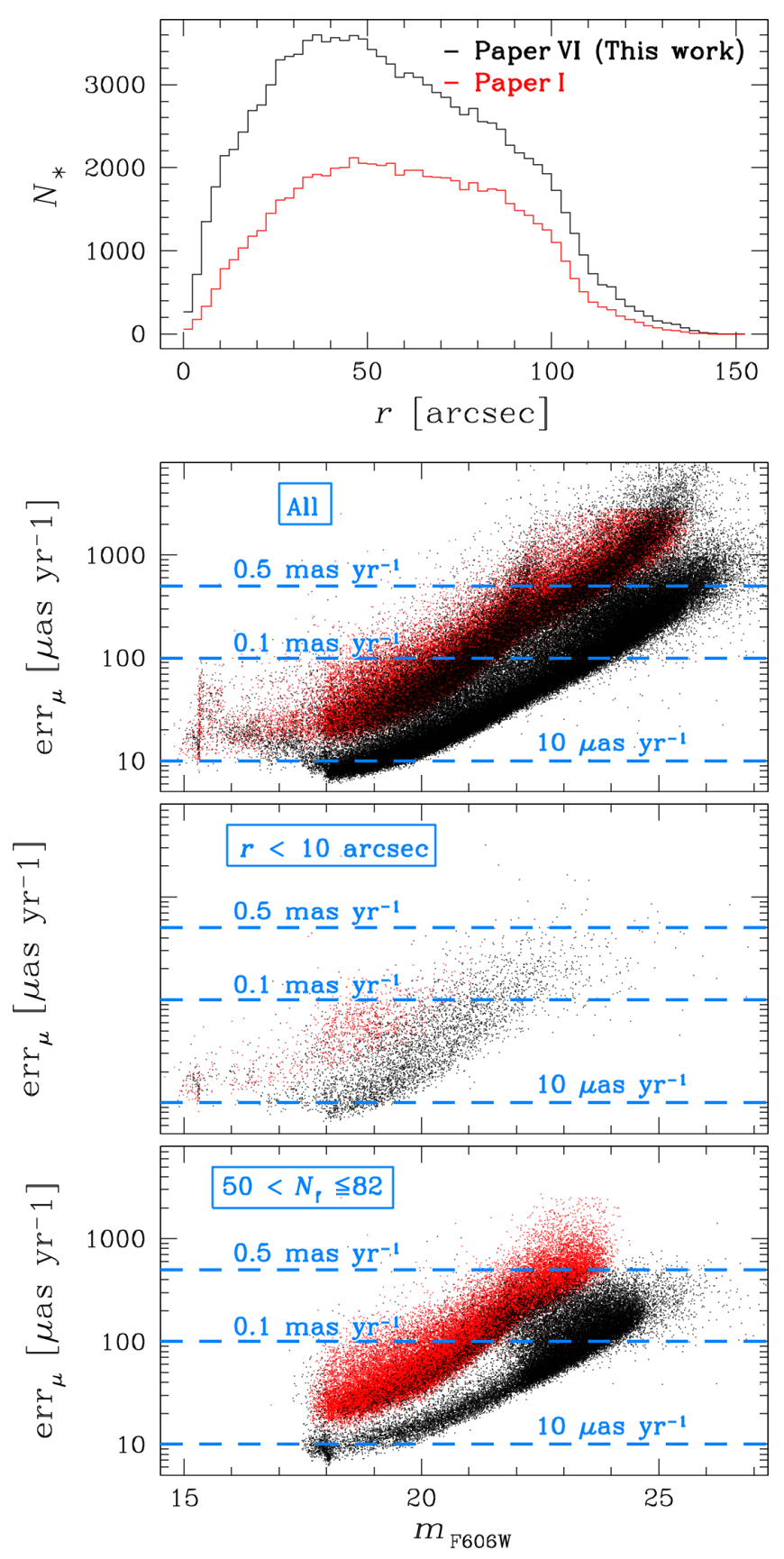

Figure 18. Comparison between the PMs obtained in this paper (Paper VI, in black) and in Paper I (in red). The two histograms in the top panel show the number of sources as a function of distance from the cluster center. Overall, our new catalog contains more sources. In the three remaining panels, we compare the PM errors. From top to bottom, we show all stars, stars within 10 arcsec from the center of NGC 362, and stars with PM obtained by using between 50 and 82 exposures, respectively. 
meaning that stars at the same magnitude are measured with about the same precision, regardless of the crowding.

These examples summarize some of the differences between first- and second-pass photometry. While the position of very-bright stars can be measured reasonably well even in very-crowded environments without neighbor subtraction, the fainter the stars and the more crowded the field, the more important the neighbor subtraction becomes. Furthermore, KS2 allows us to find and measure stars as faint as $m_{\mathrm{F} 606 \mathrm{~W}} \sim 27$, two magnitudes fainter than in the old-PM catalog.

\section{SMC}

The absolute PM of the SMC was computed by using the absolute PM value of NGC 362 in Sect. 3.6. We calculated the $4 \sigma$-clipped median value of the relative PM of SMC stars along each direction. We estimate:

$$
\begin{aligned}
& \left(\mu_{\alpha} \cos \delta, \mu_{\delta}\right)_{\mathrm{SMC} @ \mathrm{NGC} 362, \text { relative }} \\
& =(-5.913 \pm 0.008,1.291 \pm 0.007) \mathrm{mas} \mathrm{yr}^{-1}
\end{aligned}
$$

thus resulting in:

$$
\begin{aligned}
& \left(\mu_{\alpha} \cos \delta, \mu_{\delta}\right)_{\mathrm{SMC} @ N G C} 362, \text { absolute } \\
& =(0.790 \pm 0.279,-1.116 \pm 0.135) \text { mas } \mathrm{yr}^{-1}
\end{aligned}
$$

Error bars in the latter PM values are the sums in quadrature of the errors of the absolute PM of NGC 362 and of the relative bulk PM of SMC stars in our field. The expected contribution of the parallax (see discussion in Sect. 3.6) for the stars in our FoV and with the temporal coverage of our observations is of $10 \mu \mathrm{as} \mathrm{yr}^{-1}$ along $\mu_{\delta}$, while the size of the effect along $\mu_{\alpha} \cos \delta$ is negligible. The median PM error of the SMC stars used in

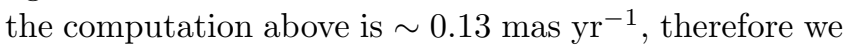
chose to not correct for the parallax effects. In the top panel of Fig. 19, we show the absolute-PM VPD with the absolute motion of the SMC highlighted. In the bottom panel of the same Figure, we present a comparison with the absolute PM of the SMC from the literature.

The measured PM of SMC stars in the NGC 362 field is not an unbiased estimate of the PM of the SMC COM. Depending on where one points in the SMC, different components of the $3 \mathrm{D} \mathrm{COM}$ velocity vector project onto the local line of sight, West, and North directions (van der Marel et al. 2002). After correcting for viewing perspective as in van der Marel \& Guhathakurta (2008), our new measurement becomes:

$$
\begin{aligned}
& \left(\mu_{\alpha} \cos \delta, \mu_{\delta}\right)_{\mathrm{SMC} @ \mathrm{COM}, \text { absolute }} \\
& =(0.741 \pm 0.279,-1.135 \pm 0.135) \text { mas } \mathrm{yr}^{-1}
\end{aligned}
$$

Here the position of the SMC COM is assumed to be at the photometric centroid of the old stars in the SMC. Note that the viewing perspective correction is significantly smaller than the random errors in our measurement.

If one assumes for simplicity that there are no internal motions within the SMC, then this is an estimate for the $\mathrm{PM}$ of the SMC COM. This result can be compared to existing estimates for the PM of the SMC COM. van der Marel \& Sahlmann (2016) presented results based on a combination of HST measurements of five fields centered on background quasars from Kallivayalil et al. (2013) and the Gaia DR1 Tycho-Gaia Astrometric Solution (TGAS) catalog measurements of 8 supergiant stars. The results depend on the exact position of the COM, and the assumed SMC internal kinematics. But with the same assumptions for these as above, they found that $\left(\mu_{\alpha} \cos \delta, \mu_{\delta}\right)_{\mathrm{SMC}}=(0.740 \pm 0.072,-1.202 \pm 0.1070$ mas $\mathrm{yr}^{-1}$. Therefore, our new data are consistent with existing knowledge of the PM of SMC COM, and they do not significantly improve the existing uncertainties.

Conversely, one could assume that the SMC COM PM is already known from the literature, and then use our measurement to determine the internal PM kinematics of the SMC at the position of NGC 362. This yields:

$$
\begin{aligned}
& \left(\mu_{\alpha} \cos \delta, \mu_{\delta}\right)_{\mathrm{SMC}, \text { int }} \\
& =(0.001 \pm 0.288,0.067 \pm 0.172) \mathrm{mas} \mathrm{yr}^{-1}
\end{aligned}
$$

Given the SMC distance of $\sim 62.8 \mathrm{kpc}$, this differs from zero by $20 \mathrm{~km} \mathrm{~s}^{-1}$, but this is well within the uncertainties of $86 \mathrm{~km} \mathrm{~s}^{-1}$ and $51 \mathrm{~km} \mathrm{~s}^{-1}$, respectively. The measurement uncertainties are insufficient to probe the internal PM kinematics at interesting levels. For comparison, the known internal line-of-sight motions of different stellar populations in the SMC have values $|\Delta V| \lesssim 30$ $\mathrm{km} \mathrm{s}^{-1}$ (Dobbie et al. 2014).

\section{THE ELECTRONIC CATALOG}

The first 10 lines of the astrometric catalog of NGC 362 used in this paper are presented in Table 2. The catalog contains both the original and the corrected PMs. The $X$ and $Y$ positions in our reference frame (columns 3 and 4) increase toward West and North, respectively. The pixel scale of our reference frame is set to 40 mas pixel ${ }^{-1}$ (see Appendix A). The $\chi_{\mathrm{X}}^{2}$ and $\chi_{\mathrm{Y}}^{2}$ values (columns 9 and 10) are reduced $\chi^{2}$. The initial $\left(N_{\mathrm{f}}\right)$ and final $\left(N_{\mathrm{u}}\right)$ number of images considered in the fit of the PMs are shown in columns (11) and (12). Column (13) lists the temporal baseline (in yr) adopted to compute the PMs. The "ID" column presents the IDs of the reduction process. 

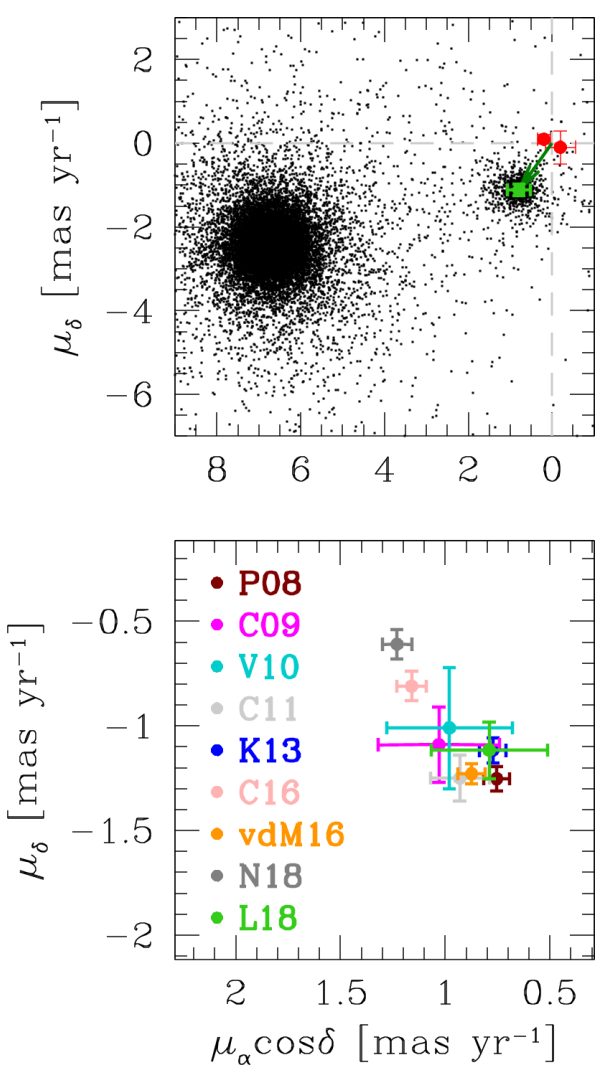

Figure 19. (Top): VPD of absolute PMs in equatorial coordinates. Red points are the galaxies used to compute the absolute PM as in Fig. 16. The SMC absolute motion relative to the galaxies and the absolute PM value of the SMC are depicted in dark and light green, respectively. (Bottom): Comparison between our estimate of the absolute PM of the SMC (L18, green) and literature values: Piatek et al. (2008, P08, brown), Costa et al. (2009, C09, magenta), Vieira et al. (2010, V10, turquoise), Costa et al. (2011, C11, light gray), Kallivayalil et al. (2013, K13, blue), Cioni et al. (2016, C16, pink), van der Marel \& Sahlmann (2016, vdM16, orange), Niederhofer et al. (2018, N18, dark gray).

The first 10 lines of the photometric catalogs of filter F606W and F814W are shown in Table 3 and Table 4, respectively. Magnitudes in column (1) are in the Vegamag system. The zero-point to subtract to column (1) to obtain the instrumental magnitudes is 31.7878 and 31.0204 for F606W- and F814W-filter magnitudes, respectively. 


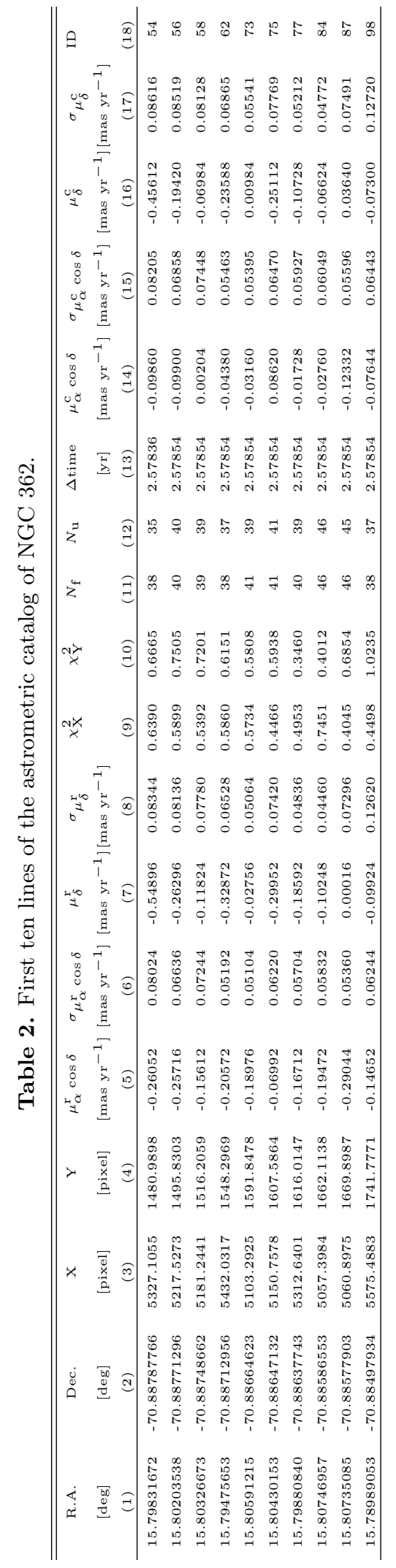

Table 3. First ten lines of the photometric catalog of NGC 362 for filter F606W.

\begin{tabular}{cccccrrr}
\hline \hline$m$ & RMS & QFIT & $o$ & \multicolumn{1}{c}{ RADXS } & $N_{\mathrm{f}}$ & $N_{\mathrm{u}}$ & RMS sky in counts \\
\hline 19.5180 & 0.1265 & 0.999 & 0.00051 & 0.0009 & 1 & 1 & 235.91 \\
19.4744 & 0.0030 & 1.000 & 0.00068 & -0.0034 & 2 & 2 & 268.41 \\
19.4463 & 0.0025 & 1.000 & 0.00002 & 0.0013 & 2 & 2 & 282.98 \\
18.8895 & 0.0727 & 1.000 & 0.00032 & 0.0007 & 1 & 1 & 352.13 \\
18.4073 & 0.0151 & 1.000 & 0.00000 & 0.0019 & 3 & 3 & 636.99 \\
19.6737 & 0.0069 & 1.000 & 0.00001 & -0.0021 & 3 & 3 & 199.27 \\
19.1991 & 0.0087 & 0.999 & 0.00025 & 0.0043 & 2 & 2 & 292.53 \\
19.2239 & 0.0158 & 1.000 & 0.00734 & 0.0013 & 4 & 4 & 294.71 \\
19.5399 & 0.0192 & 1.000 & 0.01133 & -0.0010 & 4 & 4 & 260.50 \\
19.7746 & 0.1579 & 0.999 & 0.00000 & 0.0029 & 1 & 1 & 178.40 \\
\hline
\end{tabular}

Note-The parameter $r_{\mathrm{N}}$ described in Appendix A can be computed as the ratio between the number of individual exposures used to measure a stellar position and flux $\left(N_{\mathrm{u}}\right)$ and the total number of exposures that star was actually found $\left(N_{\mathrm{f}}\right)$.

Table 4. First ten lines of the photometric catalog of NGC 362 for filter F814W.

\begin{tabular}{ccccrrrr}
\hline \hline$m$ & RMS & QFIT & $o$ & \multicolumn{1}{c}{ RADXS } & $N_{\mathrm{f}}$ & $N_{\mathrm{u}}$ & RMS sky in counts \\
\hline 19.0207 & 0.1598 & 1.000 & 0.00045 & 0.0026 & 1 & 1 & 189.99 \\
18.9887 & 0.0072 & 1.000 & 0.00102 & -0.0003 & 2 & 2 & 223.30 \\
18.9464 & 0.0040 & 1.000 & 0.00004 & 0.0014 & 2 & 2 & 217.67 \\
18.4258 & 0.0952 & 1.000 & 0.00060 & -0.0024 & 1 & 1 & 362.10 \\
17.9270 & 0.0074 & 1.000 & 0.00001 & 0.0006 & 3 & 3 & 564.56 \\
19.1586 & 0.0092 & 1.000 & 0.00005 & -0.0005 & 3 & 3 & 177.26 \\
18.7183 & 0.0075 & 1.000 & 0.00043 & 0.0015 & 2 & 2 & 252.12 \\
18.7398 & 0.0175 & 0.999 & 0.00940 & 0.0007 & 4 & 4 & 243.20 \\
19.0367 & 0.0126 & 1.000 & 0.01371 & -0.0010 & 4 & 4 & 210.64 \\
19.2601 & 0.1958 & 1.000 & 0.00000 & 0.0027 & 1 & 1 & 163.91 \\
\hline
\end{tabular}

